\title{
OPERATIONS ON ARC DIAGRAMS AND DEGENERATIONS FOR INVARIANT SUBSPACES OF LINEAR OPERATORS
}

\author{
JUSTYNA KOSAKOWSKA AND MARKUS SCHMIDMEIER \\ Dedicated to Professor Daniel Simson on the occasion of his 70th birthday
}

\begin{abstract}
We study geometric properties of varieties associated with invariant subspaces of nilpotent operators. There are algebraic groups acting on these varieties, and we give dimensions of orbits of these actions. Moreover, a combinatorial characterization of the partial order given by degenerations is described.
\end{abstract}

\section{INTRODUCTION}

Arc diagrams represent the isomorphism types of certain invariant subspaces of nilpotent linear operators, which in turn correspond to the orbits of the action of an algebraic group on the representation space. We show that operations on arc diagrams provide a combinatorial description for the degeneration order given by this group action.

1.1. Operations on arc diagrams. Two diagrams of arcs and poles are said to be in arc order if the first is obtained from the second by a sequence of moves of type
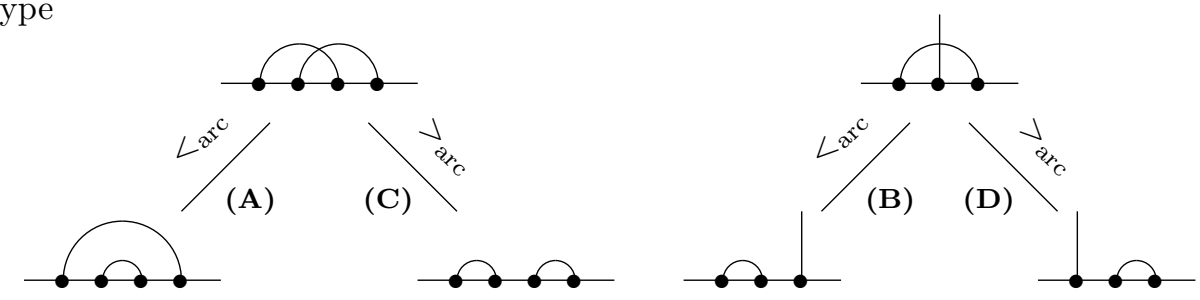

If the arc diagrams $\Delta$ and $\Delta^{\prime}$ are in relation, we write $\Delta \leq \operatorname{arc} \Delta^{\prime}$. We ask:

Q1: For two arc diagrams, decide if they are in arc order.

Q2: If two arc diagrams are in arc order, determine a sequence of moves which transforms one into the other.

Formally, an arc diagram is a finite set of arcs and poles in the Poincaré half plane. We assume that all end points are natural numbers (arranged from right to left) and permit multiple arcs and poles.

Received by the editors February 13, 2012 and, in revised form, August 16, 2012 and June 4, 2013.

2010 Mathematics Subject Classification. Primary 14L30, 16G20; Secondary 16G70, 05C85, $47 \mathrm{~A} 15$.

Key words and phrases. Degenerations, partial orders, Hall polynomials, nilpotent operators, invariant subspaces, Littlewood-Richardson tableaux.

The first named author was partially supported by Research Grant No. DEC2011/02/A/ST1/00216 of the Polish National Science Center.

(C) 2014 American Mathematical Society Reverts to public domain 28 years from publication 
Example. The diagrams $\Delta$ and $\Delta^{\prime}$ are in arc order via a single move of type (B).

$\Delta:$

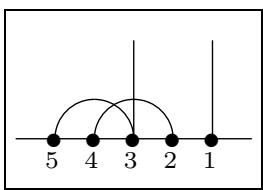

$\Delta^{\prime}:$

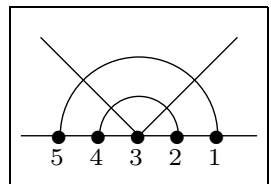

1.2. Short exact sequences of linear operators. Let $k$ be a field. We call a $k[T]$-module $N_{\alpha}=N_{\alpha}(k)=\bigoplus_{i=1}^{s} k[T] /\left(T^{\alpha_{i}}\right)$, where $\alpha=\left(\alpha_{1} \geq \cdots \geq \alpha_{s}\right)$ is a partition, a nilpotent linear operator. A monomorphism between two nilpotent linear operators is an embedding of an invariant subspace. Given three partitions $\alpha, \beta, \gamma$, we consider the subset $V_{\alpha, \gamma}^{\beta}(k)$ of $H_{\alpha}^{\beta}(k)=\operatorname{Hom}_{k}\left(N_{\alpha}, N_{\beta}\right)$ of all embeddings $f: N_{\alpha} \rightarrow N_{\beta}$ of $k[T]$-modules which give rise to a short exact sequence

$$
0 \longrightarrow N_{\alpha} \stackrel{f}{\longrightarrow} N_{\beta} \longrightarrow N_{\gamma} \longrightarrow 0 .
$$

Suppose now that the field $k$ is algebraically closed. Then the subset $V_{\alpha, \gamma}^{\beta}(k) \subset$ $H_{\alpha}^{\beta}(k)$ is constructible and there is an algebraic group acting on it such that the isomorphism classes of short exact sequences are in one-to-one correspondence with the orbits under this group action.

For points $Y=\left(N_{\alpha}, N_{\beta}, f\right), Z=\left(N_{\alpha}, N_{\beta}, g\right)$ in $V_{\alpha, \gamma}^{\beta}$, we define $Y \leq_{\operatorname{deg}} Z$ if $g$ occurs in the closure of the orbit $G_{f}$ of $f$ under this group action. We ask:

Q3: Given two embeddings $Y, Z \in V_{\alpha, \gamma}^{\beta}$, does the relation $Y \leq_{\operatorname{deg}} Z$ hold?

Q4: If $Y \leq_{\operatorname{deg}} Z$, can we find a sequence $Y=Y_{0} \leq_{\operatorname{deg}} Y_{1} \leq_{\operatorname{deg}} \cdots \leq_{\operatorname{deg}} Y_{s}=Z$ such that the dimensions of two subsequent orbits differ by one?

It is the aim of this paper to shed light on how Q1 and Q3 are related, and to provide an algorithm for Q2 which in turn yields a critereon for Q4.

1.3. From short exact sequences to arc diagrams. In case the partition $\alpha$ has first (or equivalently all) entries at most 2, the isomorphism types of embeddings in $V_{\alpha, \gamma}^{\beta}$ are determined combinatorially:

Proposition 1.1 ([17, Proposition 2]). Let $k$ be any field. For partitions $\alpha, \beta, \gamma$ with $\alpha_{1} \leq 2$, there is a one-to-one correspondence

$$
\left\{\text { embeddings in } V_{\alpha, \gamma}^{\beta}(k)\right\} / \cong \stackrel{1-1}{\longleftrightarrow}\{\text { Klein tableaux of type }(\alpha, \beta, \gamma)\} \text {. }
$$

A Klein tableau of type $(\alpha, \beta, \gamma)$ is a skew diagram of shape $\beta \backslash \gamma$ with $\bar{\alpha}_{1}$ symbols 1 and $\bar{\alpha}_{2}$ entries $2_{\mathrm{r}}$ for suitable subscripts $r$; see Section 2.5 . Here $\bar{\alpha}$ denotes the conjugate of $\alpha$, so the condition $\alpha_{1} \leq 2$ implies that all entries in the tableau are at most 2 .

A Klein tableau $\Pi$ determines an arc diagram, as follows. Suppose $\Pi$ has $e=\beta_{1}$ rows.

- Arrange the vertices $e, e-1, \ldots, 1$ on a horizontal line.

- For each symbol $2 \mathrm{r}$ in row $m$, draw an arc above the line connecting $m$ with $r$.

- If the number of arcs ending at $r$ is less than the number of symbols 1 in row $r$, draw for each remaining symbol a vertical line above $r$. 
Example. The Klein tableau $\Pi$ has the arc diagram $\Delta$.

$\Pi$ :

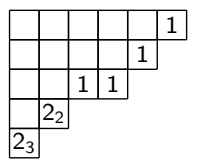

$\Delta:$

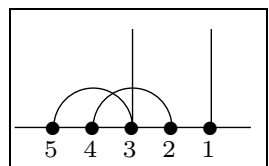

Definition. The arc diagram $\Delta(Y)$ of an invariant subspace $Y \in V_{\alpha, \gamma}^{\beta}$ is defined to be the arc diagram given by the Klein tableau representing the isomorphism class of $Y$ in $V_{\alpha, \gamma}^{\beta}$. We say two invariant subspaces $Y, Z \in V_{\alpha, \gamma}^{\beta}$ are in arc order, in symbols $Y \leq_{\operatorname{arc}} Z$, if $\Delta(Y) \leq_{\operatorname{arc}} \Delta(Z)$ holds.

1.4. Main results. We can now relate the above problems $\mathrm{Q} 1$ and Q3:

Theorem 1.2. Suppose that $k$ is an algebraically closed field and that $\alpha, \beta, \gamma$ are partitions with $\alpha_{1} \leq 2$. For invariant subspaces $Y, Z \in V_{\alpha, \gamma}^{\beta}$ we have

$$
Y \leq_{\operatorname{deg}} Z \quad \text { if and only if } \quad Y \leq_{\operatorname{arc}} Z .
$$

In the proof of the "only if" part, we will present an algorithm which delivers a sequence of arc moves that convert $Z$ to $Y$, addressing Q2.

The diagram of an invariant subspace determines the dimension of its orbit in $V_{\alpha, \gamma}^{\beta}$ as follows. For a partition $\lambda$, the length is given by $|\lambda|=\lambda_{1}+\lambda_{2}+\cdots$, and the moment is $n(\lambda)=\sum_{i} \lambda_{i}(i-1)$.

Theorem 1.3. Let $k$ be an algebraically closed field, and let $\alpha, \beta, \gamma$ be partitions. Suppose the arc diagram $\Delta$ of an invariant subspace $Y=\left(N_{\alpha}, N_{\beta}, f\right) \in V_{\alpha, \gamma}^{\beta}(k)$ has $x(\Delta)$ crossings. Then

$$
\operatorname{dim} G_{f}=\operatorname{deg} h_{\alpha, \gamma}^{\beta}+\operatorname{deg} a_{\alpha}-x(\Delta),
$$

where $\operatorname{deg} h_{\alpha, \gamma}^{\beta}=n(\beta)-n(\alpha)-n(\gamma)$ is the degree of the Hall polynomial $h_{\alpha, \gamma}^{\beta}(q)$ and $\operatorname{deg} a_{\alpha}=|\alpha|+2 n(\alpha)$ is the degree of the polynomial $a_{\alpha}(q)$ which counts the automorphisms of $N_{\alpha}\left(\mathbb{F}_{q}\right)$.

As a consequence we obtain a critereon for Q4: Assume $Z$ can be converted to $Y$ via a sequence of arc moves such that each move reduces the number of crossings by 1 . Then there exists a sequence of orbits in $V_{\alpha, \gamma}^{\beta}$ such that in each step the dimension increases by 1 , and conversely. The first example in Section 5.3 shows that in general the answer to Q4 is No (consider the Klein tableaux $\Pi_{7}$ and $\Pi_{5}$, or $\Pi_{6}$ and $\left.\Pi_{4}\right)$.

1.5. Diagrams of oriented arcs. Note that the arc moves (A) and (B) share the property that at each vertex, the number of incoming arcs and the number of outgoing arcs remain constant, where poles are considered as incoming. It turns out that the question of whether two arc diagrams are in arc order via moves of type (A) or (B) has an interpretation in terms of linear operators.

Recall the Theorem by Green and Klein, which we present in the version for linear operators.

Theorem 1.4 ([7]). Let $k$ be any field, and let $\alpha, \beta, \gamma$ be partitions. There exists a short exact sequence of linear operators

$$
0 \longrightarrow N_{\alpha} \longrightarrow N_{\beta} \longrightarrow N_{\gamma} \longrightarrow 0
$$

if and only if there exists a Littlewood-Richardson (LR-)tableau of type $(\alpha, \beta, \gamma)$. 
We will consider LR-tableaux in Section 2.1 for the purpose of this paragraph, an LR-tableau $\Gamma$ is just a Klein tableau $\Pi$ with all subscripts omitted. We call $\Gamma$ the underlying LR-tableau of $\Pi$, and $\Pi$ a refinement of $\Gamma$. Suppose an arc diagram $\Delta$ is given by a Klein tableaux $\Pi$. Observe that exactly the arc moves of type (A) and (B) are given by changing subscripts in the Klein tableau, and hence leave the underlying LR-tableau unchanged.

Suppose $\Gamma$ is an LR-tableau of type $(\alpha, \beta, \gamma)$. Denote by $V_{\Gamma}$ the constructible subset of $H_{\alpha}^{\beta}$ (contained in $V_{\alpha, \gamma}^{\beta}$ ) of all invariant subspaces $Y$ for which the Klein tableau representing the isomorphism type of $Y$ is a refinement of $\Gamma$.

Theorem 1.5. Suppose $k$ is an algebraically closed field, and $\Gamma$ is an LR-tableau with entries at most 2. For invariant subspaces $Y, Z \in V_{\Gamma}$ we have $Y \leq \operatorname{deg} Z$ if and only if $Y \leq_{\operatorname{arc}} Z$. If one of the relations holds, then $Y$ is obtained from $Z$ by a sequence of arc moves of type (A) and (B).

1.6. History and related results. On the sets of orbits in $V_{\alpha, \gamma}^{\beta}(k)$ we consider the partial order $\leq_{\text {deg }}$ given by degenerations and the partial orders $\leq_{\text {ext }}$, $\leq_{\text {hom }}$ (see Section 3). It is well known that

$$
\leq_{\text {ext }} \Longrightarrow \leq_{\text {deg }} \Longrightarrow \leq_{\text {hom }}
$$

(see [2], 13]). But the implication $\leq_{\text {hom }} \Longrightarrow \leq_{\text {ext }}$ is not always true. There is an open problem to find classes of algebras or modules for which the last implication holds. This is the case for representations of Dynkin and extended Dynkin quivers ([2], 3], [19]), for representation directed algebras ([2]), and for posets of finite prinjective type $([9)$. For more comprehensive information about degenerations of modules we refer the reader to [2, 3, 13 .

The problem of classifying invariant subspaces, up to isomorphism, has been studied since Birkhoff [1], where he challenged us to classify all embeddings of a subgroup in an abelian group, up to automorphisms of the ambient group. There are many variations of the original problem, as one can take for the coefficient ring any local uniserial ring, and as one can admit several submodules. For a detailed investigation of the representation theoretic complexity of many categories of poset representations with coefficients in a local uniserial ring, we refer the reader to [18.

Here we are interested in the category $\mathcal{S}_{2}(k)$ of all embeddings $f: N_{\alpha} \rightarrow N_{\beta}$ where $\alpha, \beta$ are partitions such that $\alpha_{1} \leq 2$. This is a full subcategory, closed under subobjects, of the category of representations of the one point poset with coefficients in the local uniserial ring $k[[T]]$. The category $\mathcal{S}_{2}(k)$ is of discrete representation type, but not representation directed.

1.7. Organization of this paper. In Section 2 we give definitions and notation concerning LR-tableaux, Klein tableaux, arc diagrams and the category $\mathcal{S}_{2}(k)$. In the proof of Lemma 2.2 we show how a formula by T. Klein in [8] computes the number $x(\Delta)$ of crossings in an arc diagram $\Delta$ as the deviation $x(\Pi)$ from dominance of the underlying Klein tableau.

The partial orders $\leq_{\text {arc }}, \leq_{\text {ext }}, \leq_{\text {deg }}$ and $\leq_{\text {hom }}$ are introduced in Section 3 , their relation is discussed in Theorem 3.4 which also deals with the case where the base field is not algebraically closed. We show the following implications:

$$
\leq_{\text {ext }} \Longrightarrow \leq_{\text {deg }} \Longrightarrow \leq_{\text {hom }} .
$$


In Section 4 we complete the proofs of Theorems 1.2 and 1.5 by showing the implications

$$
\leq_{\text {hom }} \Longrightarrow \leq_{\text {arc }} \Longrightarrow \leq_{\text {ext }} .
$$

For the first, we present an algorithm which determines for given objects $Y, Z \in \mathcal{S}_{2}$ satisfying $Y \leq_{\text {hom }} Z$ a sequence of arc operations that transform the arc diagram for $Z$ into the arc diagram for $Y$.

Let $\Pi$ be a Klein tableau, $q$ a prime power and $k$ the algebraic closure of $\mathbb{F}_{q}$. Denote by $g_{\Pi}(q)$ the number of monomorphisms in $V_{\alpha, \gamma}^{\beta}\left(\mathbb{F}_{q}\right)$ corresponding to $\Pi$, and let $Y=\left(N_{\alpha}, N_{\beta}, f\right)$ be a monomorphism in $V_{\alpha, \gamma}^{\beta}(k)$, also corresponding to $\Pi$. In Section [5] we use a result by Lang and Weil [11 which links the dimension of the variety $G_{f}(k)$ to the degree of the polynomial $g_{\Pi}(q)$, and hence to the degree of the Hall polynomial $h_{\alpha, \gamma}^{\beta}(q)$, the cardinality $a_{\alpha}(q)$ of the automorphism group of $N_{\alpha}\left(\mathbb{F}_{q}\right)$ and the number of crossings $x(\Delta)$ of the arc diagram given by $\Pi$.

In order to apply this result, we verify in Lemma 5.1 that the category $\mathcal{S}_{2}$ can be defined over $\mathbb{Z}$. This finishes the proof of Theorem 1.3 . Finally, in Theorem 5.7 we describe the minimal and the maximal elements in the partially ordered set of all arc diagrams corresponding to a given partition type.

\section{Notation AND DEFinitions}

We introduce Klein tableaux, LR-tableaux and arc diagrams, as they provide isomorphism invariants for the objects in the category $\mathcal{S}_{2}$.

2.1. From tableaux to arc diagrams. For a partition $\alpha$ we denote by $\bar{\alpha}$ the conjugate, so $\alpha_{i}$ is the length of the $i$-th column and $\bar{\alpha}_{j}$ the length of the $j$-th row of the corresponding Young diagram. The sum of the entries of $\alpha$ is denoted by $|\alpha|$.

Definition. (1) Given three partitions $\alpha, \beta, \gamma$, an LR-tableau of type $(\alpha, \beta, \gamma)$ is a skew diagram of shape $\beta \backslash \gamma$ with $\bar{\alpha}_{1}$ entries $1, \bar{\alpha}_{2}$ entries 2 , etc. The entries are weakly increasing in each row, strictly increasing in each column, and satisfy the lattice permutation property (for each $c \geq 0, \ell \geq 2$ there are at least as many entries $\ell-1$ on the right hand side of the $c$-th column as there are entries $\ell$ ).

(2) A Klein tableau of type $(\alpha, \beta, \gamma)$ is an LR-tableau of the same type where in addition each entry $\ell \geq 2$ carries a subscript, subject to the conditions (see [8, (1.2)]):

(a) If a symbol $\ell_{r}$ occurs in the $m$-th row in the tableau, then $1 \leq r \leq$ $m-1$.

(b) If $\ell_{\mathrm{r}}$ occurs in the $m$-th row and the entry above $\ell_{\mathrm{r}}$ is $\ell-1$, then $r=m-1$.

(c) The total number of symbols $\ell_{r}$ in the tableau cannot exceed the number of entries $\ell-1$ in row $r$.

(3) Given a Klein tableau $\Pi$, we obtain the underlying LR-tableau $\Gamma$ by omitting the subscripts of those entries. We say that $\Pi$ is a refinement of $\Gamma$.

(4) From an LR-tableau $\Gamma$ one can obtain a Klein tableau $\Pi$ by working through the entries in $\Gamma$ row by row (starting at the top) and assigning to each symbol $\ell \geq 2$ the largest available subscript (due to the lattice permutation property, there is always a subscript available). Then $\Pi$ is the dominant Klein tableau refining $\Gamma$. 
We have seen in Section 1.3 how a Klein tableau determines an arc diagram. The following result follows immediately.

Lemma 2.1. Suppose the Klein tableau $\Pi$ is a refinement of the LR-tableau $\Gamma$ and has arc diagram $\Delta$.

(1) At a vertex $m$ in $\Delta$, the number of incoming arcs plus the number of poles can be read as the number of entries 1 in row $m$ in $\Gamma$ or in $\Pi$.

(2) For each vertex $m$ in $\Delta$, the number of outgoing arcs equals the number of 2 's in row $m$ in $\Gamma$.

(3) Each box $[2$ in row $m$ in $\Pi$ corresponds to an arc from $m$ to $r$ in $\Delta$, and conversely.

(4) A Klein tableau of given type $(\alpha, \beta, \gamma)$ is determined uniquely by its arc diagram.

(5) The arc diagram associated with a dominant Klein tableau has no intersections.

Suppose that $\Pi$ and $\Pi^{\prime}$ are two Klein tableaux of partition type $(\alpha, \beta, \gamma)$ and that they are represented by arc diagrams $\Delta$ and $\Delta^{\prime}$, respectively. We define $\Pi \leq$ arc $\Pi^{\prime}$ if $\Delta \leq_{\text {arc }} \Delta^{\prime}$. It follows from the lemma that the dominant Klein tableaux are minimal with respect to this relation.

2.2. A formula in Klein's paper. With a given LR-tableau $\Gamma$, we have associated the dominant Klein tableau $\Pi_{0}$; it is special among all Klein tableaux refining $\Gamma$ in the sense that the associated arc diagram has no intersections. The "distance" of an arbitrary Klein tableau $\Pi$ refining $\Gamma$ from the dominant one is introduced in [8. Definition 1.4] as the deviation $x(\Pi)$ from dominance for which the following formula is given:

$$
\begin{aligned}
x(\Pi) & =\sum_{j}\left(\bar{\zeta}_{j}-\bar{\gamma}_{j}+\left|\beta^{j-1}\right|-\left|\beta^{j}\right|\right) \sum_{k>j}\left(\bar{\beta}_{k}^{j-1}-\bar{\zeta}_{k}\right) \\
& +\sum_{j} \sum_{\ell>j}\left(\bar{\beta}_{\ell+1}^{j}-\bar{\beta}_{\ell+1}^{j-1}\right) \sum_{k=j+1}^{\ell}\left(\bar{\beta}_{k}^{j-1}-\bar{\zeta}_{k}\right) .
\end{aligned}
$$

Here, a Klein tableau $\Pi$ of type $(\alpha, \beta, \gamma)$ with entries at most 2 is encoded by a sequence of partitions

$$
\Pi=\left[\gamma ; \zeta=\beta^{0} ; \beta^{1}, \ldots, \beta^{s}=\beta\right],
$$

where $\gamma$ represents the region in the diagram $\Pi$ given by the empty boxes; $\zeta=\beta^{0}$ is the partition for the region marked off by the entries $\square$ and 11 ; and for each $j$, the partition $\beta^{j}$ represents a region in $\Pi$ given by the empty boxes, the boxes 1 and the boxes $2 \mathrm{i}_{\mathrm{i}}$ where $i \leq j$.

We can read the deviation from dominance as the number of intersections in the corresponding arc diagram:

Lemma 2.2. Let $\Pi$ be a Klein tableau with entries at most 2 . The deviation $x(\Pi)$ from dominance equals the number $x(\Delta)$ of crossings in the arc diagram $\Delta$ which corresponds to $\Pi$.

Proof. For each of the factors in the formula for $x(\Pi)$ we give an interpretation in terms of data associated with the arc diagram $\Delta$ :

In $\Pi$, the boxes 1 are located in the skew diagram $\zeta \backslash \gamma$, so the number of boxes 1 in row $j$ is given by $\bar{\zeta}_{j}-\bar{\gamma}_{j}$. Similarly, the boxes $22_{\mathrm{j}}$ are located in the skew 
diagram $\beta^{j} \backslash \beta^{j-1}$; their number is $\left|\beta^{j}\right|-\left|\beta^{j-1}\right|$. In $\Delta$, each box $22_{\mathrm{j}}$ corresponds to an arc ending at $j$, so the difference $\bar{\zeta}_{j}-\bar{\gamma}_{j}+\left|\beta^{j-1}\right|-\left|\beta^{j}\right|$ counts the number of poles at position $j$.

As for the next factor, boxes of type $2_{\mathrm{i}}$ where $i<j$ are located in the skew diagram $\beta^{j-1} \backslash \zeta$; the number of such boxes in row $k$ is $\bar{\beta}_{k}^{j-1}-\bar{\zeta}_{k}$. The sum $\sum_{k>j}\left(\bar{\beta}_{k}^{j-1}-\bar{\zeta}_{k}\right)$ taken over all $k>j$ counts the number of arcs in $\Delta$ starting on the left of $j$ and ending on the right. Thus the $j$-th term in the first sum counts the number of crossings with poles at position $j$.

Similarly, the skew diagram $\beta^{j} \backslash \beta^{j-1}$ consists exactly of the boxes $22_{\mathrm{j}}$, so the factor $\bar{\beta}_{\ell+1}^{j}-\bar{\beta}_{\ell+1}^{j-1}$, which is the number of such boxes in the $(\ell+1)$-st row, counts the arcs from $\ell+1$ to $j$.

We have already seen that $\beta^{j-1} \backslash \zeta$ is the skew diagram which consists of the boxes $22_{\mathrm{i}}$ where $i<j$. Hence the sum $\sum_{k=j+1}^{\ell}\left(\bar{\beta}_{k}^{j-1}-\bar{\zeta}_{k}\right)$ counts the arcs which start in the interval $[j+1, \ell]$ and end in $[1, j-1]$. Those are the arcs which intersect with the arc from $\ell+1$ to $j$, and are on the right hand side of it. In conclusion, the term corresponding to $j$ and $\ell$ in the second sum counts the number of intersections of arcs from $\ell+1$ to $j$ with arcs which are on the right hand side of it.

We have seen that the first sum counts the intersections between arcs and poles in $\Delta$, while the second sum counts the intersections of arcs with arcs.

2.3. A partial ordering on LR-tableaux. We recall that there is a partial ordering on partitions given by $\zeta \leq$ part $\zeta^{\prime}$ if for each natural number $\ell$, the inequality $\sum_{i=1}^{\ell} \zeta_{i} \leq \sum_{i=1}^{\ell} \zeta_{i}^{\prime}$ holds.

Definition. Suppose $\Gamma, \Gamma^{\prime}$ are LR-tableaux of partition type $(\alpha, \beta, \gamma)$, both with entries at most 2 . Representing LR-tableaux by increasing sequences of partitions as in [12] or in the proof of Lemma 2.2 say $\Gamma=[\gamma ; \zeta ; \beta]$ and $\Gamma^{\prime}=\left[\gamma ; \zeta^{\prime} ; \beta\right]$, we define $\Gamma \leq_{\text {part }} \Gamma^{\prime}$ if $\zeta \leq_{\text {part }} \zeta^{\prime}$ holds.

This defines a partial ordering $\leq_{\text {part }}$ on the set of LR-tableaux of a given partition type $(\alpha, \beta, \gamma)$ with $\alpha_{1} \leq 2$. It is easy to see:

Lemma 2.3. Suppose $\Gamma, \Gamma^{\prime}$ are LR-tableaux of the same partition type and $\Delta, \Delta^{\prime}$ are arc diagrams of LR-type $\Gamma, \Gamma^{\prime}$, respectively.

(1) If $\Delta<_{\operatorname{arc}} \Delta^{\prime}$ via an arc operation of type (A) or (B), then $\Gamma=\Gamma^{\prime}$.

(2) If $\Delta<_{\operatorname{arc}} \Delta^{\prime}$ via an arc operation of type (C) or (D), then $\Gamma>_{\text {part }} \Gamma^{\prime}$.

2.4. Invariant subspaces. Let $k$ be an arbitrary field. For a partition $\alpha=\left(\alpha_{1} \geq\right.$ $\left.\ldots \geq \alpha_{n}\right)$ we denote by $N_{\alpha}$ or by $N_{\alpha}(k)$ the finite dimensional $k[T]$-module $N_{\alpha}(k)=$ $k[T] /\left(T^{\alpha_{1}}\right) \oplus \ldots \oplus k[T] /\left(T^{\alpha_{n}}\right)$. Note that $N_{\alpha}(k)$ can be considered as a $k[T] /\left(T^{\alpha_{1}}\right)$ module. By $\mathcal{N}$ or $\mathcal{N}(k)$ we denote this category of all nilpotent linear operators. We write the objects of $\mathcal{N}$ as pairs $(V, \varphi)$, where $V$ is the underlying $k$-vector space and $\varphi: V \rightarrow V$ the nilpotent $k$-linear endomorphism given by multiplication by $T$. If $(V, \varphi),\left(V^{\prime}, \varphi^{\prime}\right)$ are objects in $\mathcal{N}$, then a morphism $f:(V, \varphi) \rightarrow\left(V^{\prime}, \varphi^{\prime}\right)$ in $\mathcal{N}$ is a linear map $f: V \rightarrow V^{\prime}$ such that $\varphi^{\prime} f=f \varphi$.

By $\Lambda$ we denote the $k$-algebra

$$
\Lambda=\left(\begin{array}{cc}
k[T] & k[T] \\
0 & k[T]
\end{array}\right) .
$$

Let $\bmod (\Lambda)$ be the category of all finite dimensional right $\Lambda$-modules, and $\bmod _{0}(\Lambda)$ the full subcategory of $\bmod (\Lambda)$ of all modules for which the element $T=\left(\begin{array}{ll}T & 0 \\ 0 & T\end{array}\right)$ acts 
nilpotently. It is well known that objects in $\bmod _{0}(\Lambda)$ may be identified with systems $\left(N_{\alpha}, N_{\beta}, f\right)$, where $\alpha, \beta$ are partitions and $f: N_{\alpha} \rightarrow N_{\beta}$ is a $k[T]$-homomorphism. Let $A=\left(N_{\alpha}, N_{\beta}, f\right), A^{\prime}=\left(N_{\alpha^{\prime}}, N_{\beta^{\prime}}, f^{\prime}\right)$ be objects in $\bmod _{0}(\Lambda)$. A morphism $\Psi: A \rightarrow A^{\prime}$ is a pair $\left(\psi_{1}, \psi_{2}\right)$, where $\psi_{1}: N_{\alpha} \rightarrow N_{\alpha^{\prime}}, \psi_{2}: N_{\beta} \rightarrow N_{\beta^{\prime}}$ are homomorphisms of $k[T]$-modules such that $f^{\prime} \psi_{1}=\psi_{2} f$.

Denote by $\mathcal{S}$ or $\mathcal{S}(k)$ the full subcategory of $\bmod _{0}(\Lambda)$ consisting of all systems $f=\left(N_{\alpha}, N_{\beta}, f\right)$, where $f$ is a monomorphism.

For a natural number $n$, we write $\mathcal{S}_{n}$ or $\mathcal{S}_{n}(k)$ for the full subcategory of $\mathcal{S}$ of all systems where the operator acts on the subspace with nilpotency index at most $n$. Thus, the objects in $\mathcal{S}_{2}$ are the systems $\left(N_{\alpha}, N_{\beta}, f\right)$ where $\alpha_{1} \leq 2$.

2.5. Pickets and bipickets. The category $\mathcal{S}_{2}(k)$ is of particular interest for us in this paper. It has discrete representation type: Each indecomposable object is either isomorphic to a picket, that is, it has the form

$$
P_{\ell}^{m}=\left(N_{(\ell)}, N_{(m)}, \iota\right)
$$

where $0 \leq \ell \leq \min \{2, m\}$ (so the ambient space $N_{(m)}$ has only one Jordan block, and $N_{(\ell)}$ is the unique $T$-invariant subspace of dimension $\ell$ ), or to a bipicket

$$
B_{2}^{m, r}=\left(N_{(2)}, N_{(m, r)}, \delta\right)
$$

where $1 \leq r \leq m-2$ and $\delta: k[T] /\left(T^{2}\right) \rightarrow k[T] /\left(T^{m}\right) \oplus k[T] /\left(T^{r}\right)$ is given by $\delta(1)=\left(T^{m-2}, T^{r-1}\right)$. Whenever we want to emphasize the dependence on the field $k$, we will write $P_{\ell}^{m}=P_{\ell}^{m}(k)$ and $B_{2}^{m, r}=B_{2}^{m, r}(k)$.

For the definition of a Klein tableau for an object in $\mathcal{S}_{2}$ we refer to [8] or [17]; here we summarize by giving a brief description. The tableau of a picket $P_{\ell}^{m}$ consists of a single column of $m$ boxes; the $\ell$ boxes at the bottom carry the entries $1, \ldots, \ell$. In case $\ell=2$, the entry 2 has a subscript $2_{r}$ where $r=m-1$. The tableau for a bipicket $B_{2}^{m, r}$ has two columns of $m$ and $r$ boxes, which are aligned at the top. The two boxes at the bottom are $2_{\mathrm{r}}$ and 1 , respectively.

Tableaux and diagrams for the objects in ind $\mathcal{S}_{2}$

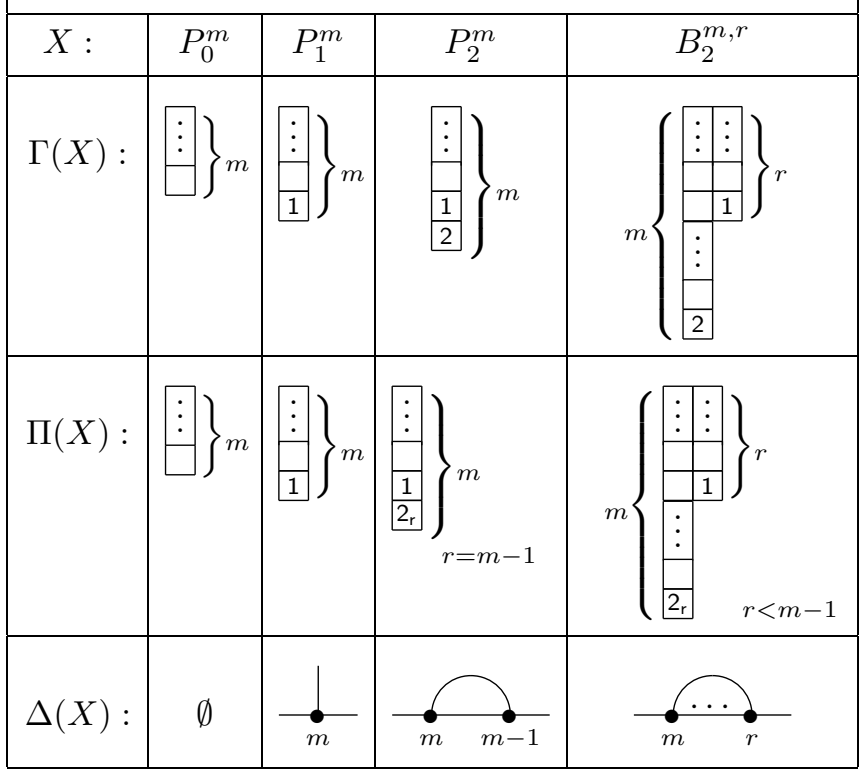


The Klein tableau for a direct sum $M \oplus M^{\prime}$ has a diagram given by the union $\beta \cup \beta^{\prime}$ of the partitions representing the ambient spaces, and in each row the entries are obtained by lexicographically ordering the entries in the corresponding rows in the tableaux for $M$ and $M^{\prime}$, with empty boxes coming first.

Example. The Klein tableaux $\Pi$ in the example in Section 1.3 is given by

$$
X=B_{2}^{5,3} \oplus B_{2}^{4,2} \oplus P_{1}^{3} \oplus P_{1}^{1} .
$$

We recall from [17, Proposition 2]:

Theorem 2.4. For any field $k$, there is a one-to-one correspondence between the isomorphism classes of objects in $\mathcal{S}_{2}(k)$ and the Klein tableaux with entries at most 2 .

The object in $\mathcal{S}_{2}(k)$ which corresponds to the Klein tableau $\Pi$ will be denoted by $M_{\Pi}$ or $M_{\Pi}(k)$. It is straightforward to recover the indecomposable summands of $M_{\Pi}$ from $\Pi$. To start, note that each entry $2_{r}$ in row $m$ gives rise to a summand of type $B_{2}^{m, r}$ if $r \leq m-2$ or $P_{2}^{m}$ if $r=m-1$.

\section{Partial orders on Klein tableaux}

For partitions $\alpha, \beta, \gamma$, denote by $\mathcal{S}_{\alpha}^{\beta}$ the full subcategory of $\mathcal{S}$ of all embeddings of type $f: N_{\alpha} \rightarrow N_{\beta}$, and by $\mathcal{S}_{\alpha, \gamma}^{\beta}$ the full subcategory of $\mathcal{S}_{\alpha}^{\beta}$ of those embeddings which satisfy in addition that Coker $f \cong N_{\gamma}$ holds.

We introduce partial orders $\leq_{\text {ext }}, \leq_{\text {deg }}, \leq_{\text {hom }}$ and $\leq_{\text {arc }}$ for objects in $\mathcal{S}_{\alpha, \gamma}^{\beta}$ and show the implications $\leq_{\text {ext }} \Longrightarrow \leq_{\text {deg }} \Longrightarrow \leq_{\text {hom }}$. Using two results from Section 4 . we can complete the proofs of Theorems 1.2 and 1.5

Whenever we deal with the degeneration order $\leq_{\mathrm{deg}}$, we will assume that $k$ is an algebraically closed field.

\subsection{Four partial orders. We consider the affine variety}

$$
H_{\alpha}^{\beta}(k)=\operatorname{Hom}_{k}\left(N_{\alpha}(k), N_{\beta}(k)\right)
$$

(consisting of all $|\beta| \times|\alpha|$-matrices with coefficients in $k$ ). On $H_{\alpha}^{\beta}(k)$ we consider the Zariski topology, and on all subsets of $H_{\alpha}^{\beta}(k)$ we work with the induced topology. Let $V_{\alpha}^{\beta}(k)$ be the subset of $H_{\alpha}^{\beta}(k)$ consisting of all matrices that define a monomorphism in the category $\mathcal{N}(k)$. Note that $V_{\alpha}^{\beta}(k)$ is a locally closed subset of $H_{\alpha}^{\beta}(k)$. On $V_{\alpha}^{\beta}(k)$ acts the algebraic group $\operatorname{Aut}_{\mathcal{N}}\left(N_{\alpha}(k)\right) \times \operatorname{Aut}_{\mathcal{N}}\left(N_{\beta}(k)\right)$ via $(g, h) \cdot f=h f g^{-1}$. Orbits of this action correspond bijectively to isomorphism classes of objects in $\mathcal{S}_{\alpha}^{\beta}$. For a map $f: N_{\alpha}(k) \rightarrow N_{\beta}(k)$, denote by $G_{f}$ the orbit of $f$ in $V_{\alpha}^{\beta}(k)$. Let $V_{\alpha, \gamma}^{\beta}(k)$ be the set of monomorphisms $f$ in $V_{\alpha}^{\beta}(k)$ such that $\left(N_{\alpha}(k), N_{\beta}(k), f\right) \in \mathcal{S}_{\alpha, \gamma}^{\beta}(k)$.

Let $Y=\left(N_{\alpha}, N_{\beta}, f\right)$ and $Z=\left(N_{\alpha}, N_{\beta}, g\right)$ be objects in $\mathcal{S}_{\alpha, \gamma}^{\beta}(k)$.

- The relation $Y \leq_{\text {ext }} Z$ holds if there exist a natural number $s$, objects $M_{i}$, $U_{i}, V_{i}$ in $\mathcal{S}(k)$ and short exact sequences $0 \rightarrow U_{i} \rightarrow M_{i} \rightarrow V_{i} \rightarrow 0$ in $\mathcal{S}(k)$ such that $Y \cong M_{1}, U_{i} \oplus V_{i} \cong M_{i+1}$ for $1 \leq i \leq s$, and $Z \cong M_{s+1}$.

- The relation $Y \leq_{\operatorname{deg}} Z$ holds if $G_{g} \subseteq \overline{G_{f}}$ in $V_{\alpha, \gamma}^{\bar{\beta}}(k)$, where $\overline{G_{f}}$ is the closure of $G_{f}$.

- The relation $Y \leq_{\text {hom }} Z$ holds if

$$
[X, Y] \leq[X, Z]
$$


for any object $X$ in $\mathcal{S}(k)$. Here we write $[X, Y]=\operatorname{dim}_{k} \operatorname{Hom}_{\Lambda}(X, Y)$ for $\Lambda$-modules $X, Y$.

- If the objects $Y, Z$ are in $\mathcal{S}_{2}$, then the relation $Y \leq_{\text {arc }} Z$ holds if $\Pi \leq$ arc $\Pi^{\prime}$, where $\Pi, \Pi^{\prime}$ are the Klein tableaux associated with $Y$ and $Z$, respectively (see Theorem 2.4).

For $\Gamma$ an LR-tableau of type $(\alpha, \beta, \gamma)$, let $V_{\Gamma}(k)$ denote the subset of $V_{\alpha, \gamma}^{\beta}(k)$ consisting of all $f$ with LR-tableau $\Gamma$ (see [17, Chapter 2]), and define $\mathcal{S}_{\Gamma}$ correspondingly. The partial orders $\leq_{\text {ext }}, \leq_{\mathrm{deg}}, \leq_{\mathrm{hom}}$, and if applicable $\leq_{\text {arc }}$, can be defined via restriction for the objects in $\mathcal{S}_{\Gamma}$.

3.2. Modules versus embeddings. The three lemmata in this section show that we can work in the module category $\bmod (\Lambda)$ in order to decide the relations $\leq_{\text {ext }}$, $\leq_{\text {deg }}$ and $\leq_{\text {hom }}$.

Lemma 3.1. The relation $Y \leq_{\text {ext }} Z$ holds if and only if there exist a natural number $s$, objects $M_{i}, U_{i}, V_{i}$ in $\bmod (\Lambda)$ and short exact sequences $0 \rightarrow U_{i} \rightarrow M_{i} \rightarrow V_{i} \rightarrow 0$ in $\bmod (\Lambda)$ such that $Y \cong M_{1}, U_{i} \oplus V_{i} \cong M_{i+1}$ for $1 \leq i \leq s$, and $Z \cong M_{s+1}$ hold.

Proof. Assume that there exist $\Lambda$-modules $M_{i}, U_{i}, V_{i}$ satisfying the required conditions. Since $M_{s+1} \cong U_{s} \oplus V_{s}, M_{s+1} \in \mathcal{S}(k)$ and since the category $\mathcal{S}(k)$ is closed under direct summands, the $\Lambda$-modules $U_{s}, V_{s}$ are in $\mathcal{S}(k)$. Moreover there exists an exact sequence

$$
0 \rightarrow U_{s} \rightarrow U_{s-1} \oplus V_{s-1} \rightarrow V_{s} \rightarrow 0
$$

and therefore $U_{s-1}, V_{s-1}$ are in $\mathcal{S}(k)$, because the category $\mathcal{S}(k)$ is closed under extensions. Continuing this way we prove that all $U_{i}, V_{i}$ are in $\mathcal{S}(k)$, and consequently $Y \leq_{\text {ext }} Z$. Since the converse implication is obvious, we are done.

Lemma 3.2. (1) For points $Y=\left(N_{\alpha}, N_{\beta}, f\right), Z=\left(N_{\alpha}, N_{\beta}, g\right)$ in $V_{\alpha, \gamma}^{\beta}(k)$, the relation $Y \leq_{\operatorname{deg}} Z$ holds if and only if $G_{g} \subseteq{\overline{G_{f}}}^{a}$, where ${\overline{G_{f}}}^{a}$ is the closure of $G_{f}$ in the variety $M_{\alpha}^{\beta}(k)$ of $\Lambda$-modules.

(2) If $\Gamma$ is an LR-tableau of type $(\alpha, \beta, \gamma)$ and $Y=\left(N_{\alpha}, N_{\beta}, f\right), Z=\left(N_{\alpha}, N_{\beta}, g\right)$ are points in $V_{\Gamma}(k)$, then the relation $Y \leq_{\operatorname{deg}} Z$ holds if and only if $G_{g} \subseteq$ ${\overline{G_{f}}}^{a}$.

Proof. We only show the first statement; the proof of the second statement is similar. The variety $M_{\alpha}^{\beta}(k)$ of $\Lambda$-modules is defined as the (closed) subset of $H_{\alpha}^{\beta}(k)$ consisting of those linear maps which define $k[T]$-homomorphisms. If $\left(N_{\alpha}, N_{\beta}, f\right) \leq_{\operatorname{deg}}$ $\left(N_{\alpha}, N_{\beta}, g\right)$, then of course $G_{g} \subseteq{\overline{G_{f}}}^{a}$ (because $\overline{G_{f}} \subseteq{\overline{G_{f}}}^{a}$ ).

Conversely, let $G_{g} \subseteq{\overline{G_{f}}}^{a}$. Since $\overline{G_{f}}=\overline{G_{f}}{ }^{a} \cap V_{\alpha, \gamma}^{\beta}(k)$ and $G_{g} \subseteq V_{\alpha, \gamma}^{\beta}(k)$ we have $G_{g} \subseteq \overline{G_{f}}$. Thus $\left(N_{\alpha}, N_{\beta}, f\right) \leq_{\operatorname{deg}}\left(N_{\alpha}, N_{\beta}, g\right)$.

Lemma 3.3. Suppose $Y, Z \in \mathcal{S}_{2}$ have the same partition type.

(1) The relation $Y \leq_{\text {hom }} Z$ holds if and only if $[X, Y] \leq[X, Z]$ holds for any object $X$ in $\mathcal{S}_{2}(k)$.

(2) The relation $Y \leq_{\text {hom }} Z$ holds if and only if $[X, Y] \leq[X, Z]$ holds for any $\Lambda$-module $X$.

Proof. We show the second statement first.

(2) It is enough to prove that if $\left[X^{\prime}, Y\right] \leq\left[X^{\prime}, Z\right]$ for any object $X^{\prime}$ in $\mathcal{S}$, then $[X, Y] \leq[X, Z]$ for any module $X$ in $\bmod (\Lambda)$. 
Let $X=\left(X_{1}, X_{2}, h\right) \in \bmod (\Lambda)$ and write $Y=\left(Y_{1}, Y_{2}, f\right)$ where $f$ is a monomorphism. Consider the object $X^{\prime}=\left(X_{1}^{\prime}, X_{2}, h^{\prime}\right) \in \mathcal{S}$, where $X_{1}^{\prime}=X_{1} /$ Ker $h$ and $h^{\prime}: X_{1}^{\prime} \rightarrow X_{2}$ is induced by $h$. Let $a=\left(a_{1}, a_{2}\right): X \rightarrow Y$ be a morphism. Note that if $x \in \operatorname{Ker} h$, then $x \in \operatorname{Ker} a_{1}$ because $f$ is a monomorphism. Therefore there exists $a_{1}^{\prime}: X_{1}^{\prime} \rightarrow Y_{1}$ such that $a_{1}=a_{1}^{\prime} \circ$ can $_{1}$, where $\operatorname{can}_{1}: X_{1} \rightarrow X_{1}^{\prime}$ is the canonical epimorphism. It is easy to see that the pair $a^{\prime}=\left(a_{1}^{\prime}, a_{2}\right)$ defines a morphism $X^{\prime} \rightarrow Y$. Writing can $=\left(\operatorname{can}_{1}, 1\right): X \rightarrow X^{\prime}$, this morphism satisfies $a=a^{\prime} \circ$ can.

Thus, can : $X \rightarrow X^{\prime}$ is a left approximation for $X$ in $\mathcal{S}$. Since can is onto, it follows that $[X, Y]=\left[X^{\prime}, Y\right]$.

(1) Next we show that if $\left[X^{\prime \prime}, Y\right] \leq\left[X^{\prime \prime}, Z\right]$ for any object $X^{\prime \prime}$ in $\mathcal{S}_{2}$, then $\left[X^{\prime}, Y\right] \leq\left[X^{\prime}, Z\right]$ for any object $X^{\prime}$ in $\mathcal{S}$.

Now let $X^{\prime}=\left(X_{1}^{\prime}, X_{2}^{\prime}, h^{\prime}\right) \in \mathcal{S}$ and $Y=\left(Y_{1}, Y_{2}, f\right) \in \mathcal{S}_{2}$, so $T^{2} Y_{1}=0$. Consider the object $X^{\prime \prime}=\left(X_{1}^{\prime \prime}, X_{2}^{\prime \prime}, h^{\prime \prime}\right) \in \mathcal{S}_{2}$, where $X_{1}^{\prime \prime}=X_{1}^{\prime} / T^{2} X_{1}^{\prime}, X_{2}^{\prime \prime}=X_{2}^{\prime} / h^{\prime}\left(T^{2} X_{1}^{\prime}\right)$ and $h^{\prime \prime}: X_{1}^{\prime \prime} \rightarrow X_{2}^{\prime \prime}$ is the map induced by $h^{\prime}$. Since $h^{\prime}$ is a monomorphism, so is $h^{\prime \prime}$. Let $\operatorname{can}=\left(\operatorname{can}_{1}, \operatorname{can}_{2}\right): X^{\prime} \rightarrow X^{\prime \prime}$ be the canonical map. We show that a morphism $a=\left(a_{1}, a_{2}\right): X^{\prime} \rightarrow Y$ factors over can. Since $T^{2} Y_{1}=0$, we have $T^{2} X_{1}^{\prime} \subseteq \operatorname{Ker} a_{1}$, so $a_{1}$ factors over $\operatorname{can}_{1}$ : Write $a_{1}=a_{1}^{\prime \prime} \circ \operatorname{can}_{1}$. Since $h^{\prime}\left(T^{2} X_{1}^{\prime}\right) \subseteq \operatorname{Ker} a_{2}$, the map $a_{2}$ factors over $\operatorname{can}_{2}: X_{2}^{\prime} \rightarrow X_{2}^{\prime \prime}$ : There is $a_{2}^{\prime \prime}$ with $a_{2}=a_{2}^{\prime \prime} \circ \operatorname{can}_{2}$. Since $\operatorname{can}_{1}$ is onto, the pair $a^{\prime \prime}=\left(a_{1}^{\prime \prime}, a_{2}^{\prime \prime}\right)$ is a morphism from $X^{\prime \prime}$ to $Y$ in $\mathcal{S}$ satisfying $a=a^{\prime \prime} \circ$ can.

We have seen that can $: X^{\prime} \rightarrow X^{\prime \prime}$ is a left approximation for $X^{\prime}$ in $\mathcal{S}_{2}$; since can is onto, it follows that $\left[X^{\prime}, Y\right]=\left[X^{\prime \prime}, Y\right]$. We are done.

3.3. The partial orders are equivalent. We can now complete the proofs of Theorem 1.2 and Theorem 1.5, up to two results about the arc order, which are shown in the next section.

We restate both theorems to include statements about arbitrary fields.

Theorem 3.4. Let $k$ be an arbitrary field and assume that $Y, Z \in \mathcal{S}_{2}(k)$ have the same partition type $(\alpha, \beta, \gamma)$. The following conditions are equivalent:

(1) $Y \leq \operatorname{arc} Z$,

(2) $Y \leq \leq_{\mathrm{ext}} Z$,

(3) $Y \leq_{\text {hom }} Z$.

If in addition the field $k$ is algebraically closed, then the conditions stated above are equivalent with

(4) $Y \leq_{\operatorname{deg}} Z$.

Proof. Applying the functor $\operatorname{Hom}_{k}(X,-)$ to the short exact sequences given in the definition of $\leq_{\text {ext }}$, it is easy to see that $Y \leq_{\text {ext }} Z$ implies $Y \leq_{\text {hom }} Z$.

If $k$ is an algebraically closed field, then by [2, 13 and Lemmata 3.2 , 3.1 and 3.3 we have

$$
Y \leq_{\mathrm{ext}} Z \Longrightarrow Y \leq_{\mathrm{deg}} Z \Longrightarrow Y \leq_{\text {hom }} Z
$$

The implications

$$
Y \leq_{\text {hom }} Z \Longrightarrow Y \leq_{\text {arc }} Z \Longrightarrow Y \leq_{\text {ext }} Z
$$

independently on $k$ follow from Theorem 4.1 and Lemma 4.3, respectively.

Corollary 3.5. Let $k$ be an arbitrary field. Suppose $Y$ and $Z$ are objects in $\mathcal{S}_{2}(k)$ corresponding to the same LR-tableau. Then

$$
Y \leq_{\text {hom }} Z \Longleftrightarrow Y \leq_{\text {arc }} Z \Longleftrightarrow Y \leq_{\text {ext }} Z \text {. }
$$


In this case it is possible to convert the arc diagram for $Z$ into the arc diagram for $Y$ using only operations of type (A) and (B).

If $k$ is an algebraically closed field, then the above conditions are equivalent to $Y \leq_{\text {deg }} Z$.

Proof. Suppose $Y, Z$ are objects in $\mathcal{S}_{2}$ corresponding to the same LR-tableau $\Gamma$. Since $Y, Z$ have the same partition type, we obtain from Theorem 5.7 that the partial orders coincide. We have seen in Lemma 2.3 that arc operations of type (C) or (D) change the LR-type of the module in one direction. As a consequence, if $Y \leq$ hom $Z$ holds, then the arc diagram for $Y$ can be obtained from the arc diagram for $Z$ by using only arc operations of type (A) or (B). In case the field $k$ is algebraically closed, the above proof where we use the second part in Lemma 3.2 shows that

$$
Y \leq_{\text {ext }} Z \Longrightarrow Y \leq_{\text {deg }} Z \Longrightarrow Y \leq_{\text {hom }} Z \text {. }
$$

\section{When ARE TWO ARC DiAgRAMS IN $\leq$ arc-RELATION?}

Our aim is to show that $Y \leq_{\text {hom }} Z$ implies $Y \leq_{\operatorname{arc}} Z$.

Theorem 4.1. Suppose the objects $Y$ and $Z$ in $\mathcal{S}_{2}(k)$ have the same partition type. If $Y \leq_{\text {hom }} Z$ holds, then so does $Y \leq \operatorname{arc} Z$.

Consider the following example:
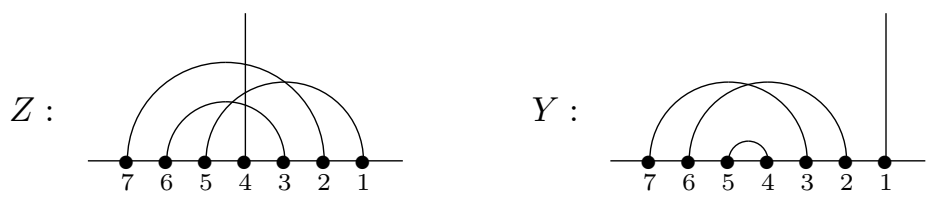

It turns out that $Y \leq_{\text {hom }} Z$. We want to show that $Y \leq_{\operatorname{arc}} Z$. In order to satisfy the condition in the definition of the $\leq_{\operatorname{arc}}$-order we need to exhibit a sequence of steps of types (A), (B), (C) and (D), which transform the arc diagram for $Y$ into the arc diagram for $Z$. This does not seem to be entirely trivial, since even inviting

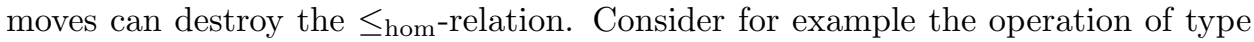
(B) which replaces the arc from 5 to 1 and the pole at 4 in $Z$ by the arc from 5 to 4 and a pole at 1 . This yields $\tilde{Z}$ :

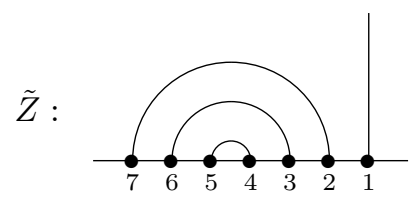

Now, however, we have gone too far as $\tilde{Z} \leq_{\text {arc }} Y$ by a move of type (A), and also $\tilde{Z} \leq_{\text {hom }} Y$ (consider the functor $\left.\operatorname{Hom}\left(B_{2}^{7,3},-\right)\right)$. We will come back to this example in Section 4.3 .

The strategy for the proof of Theorem 4.1 is to gain a thorough understanding of homomorphisms between objects in $\mathcal{S}_{2}(k)$. Recall that $Y \leq_{\text {hom }} Z$ if and only if for each indecomposable $X$, the integer

$$
\delta H(Y, Z)_{X}=[X, Z]-[X, Y]
$$


is nonnegative, where $[A, B]=[A, B]_{\mathcal{S}}=\operatorname{dim}_{k} \operatorname{Hom}_{\mathcal{S}}(A, B)$.

In the first section we will give explicit formulae for the dimensions of the homomorphism spaces between objects in $\mathcal{S}_{2}$.

Then in Section 4.2 we show how those dimensions change when $Y$ is replaced by $Z$, the module obtained from $Y$ by performing an operation of type (A), (B), (C) or (D) on the arc diagram.

We analyze the Hom-matrix $\delta H(Y, Z)$ which forms the basis of our construction of moves in the $\leq_{\text {arc }}$-direction. In particular, we can read from this matrix the multiplicities of indecomposable objects as direct summands in $Y$ and in $Z$, respectively.

The example in Section 4.3 illustrates how the decomposition of the Hom-matrix as a sum of characteristic functions of suitable parallelograms translates into the desired sequence of $\leq_{\text {arc }}$-moves.

In the last section we conclude the proof of Theorem 4.1. For this we show in Proposition 4.5 that whenever the Hom-matrix $\delta H(Y, Z)$ is nonnegative and

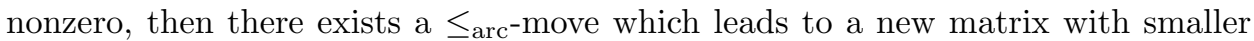
nonnegative entries.

4.1. The category $\mathcal{S}_{2}(k)$. Denote by $\mathcal{S}_{2}^{n}(k)$ the full subcategory of $\mathcal{S}_{2}(k)$ of all objects where the operator acts with nilpotency index at most $n$. We have seen in [16, Section 3.2] that $\mathcal{S}_{2}^{n}(k)$ is an exact Krull-Remak-Schmidt category with Auslander-Reiten sequences. Interestingly, the Auslander-Reiten sequences starting at a given object do not depend on the choice of $n$, provided this number is large enough, and hence are Auslander-Reiten sequences in the category $\mathcal{S}_{2}(k)$. Regarding Auslander-Reiten sequences ending at a given object, the dual result holds with the exception of the objects of type $P_{1}^{r}$ which occur as end terms of the following Auslander-Reiten sequences in $\mathcal{S}_{2}^{n}(k)$ :

$$
0 \longrightarrow B_{2}^{n, r-1} \longrightarrow B_{2}^{n, r} \oplus P_{1}^{r-1} \longrightarrow P_{1}^{r} \longrightarrow 0
$$

$(2 \leq r \leq n-2)$.

The Auslander-Reiten quiver for each of the categories $\mathcal{S}_{2}^{n}(k)$ is obtained by identifying the objects of type $P_{1}^{r}$ on the left with their counterparts on the right in the following picture, thus yielding a Moebius band:

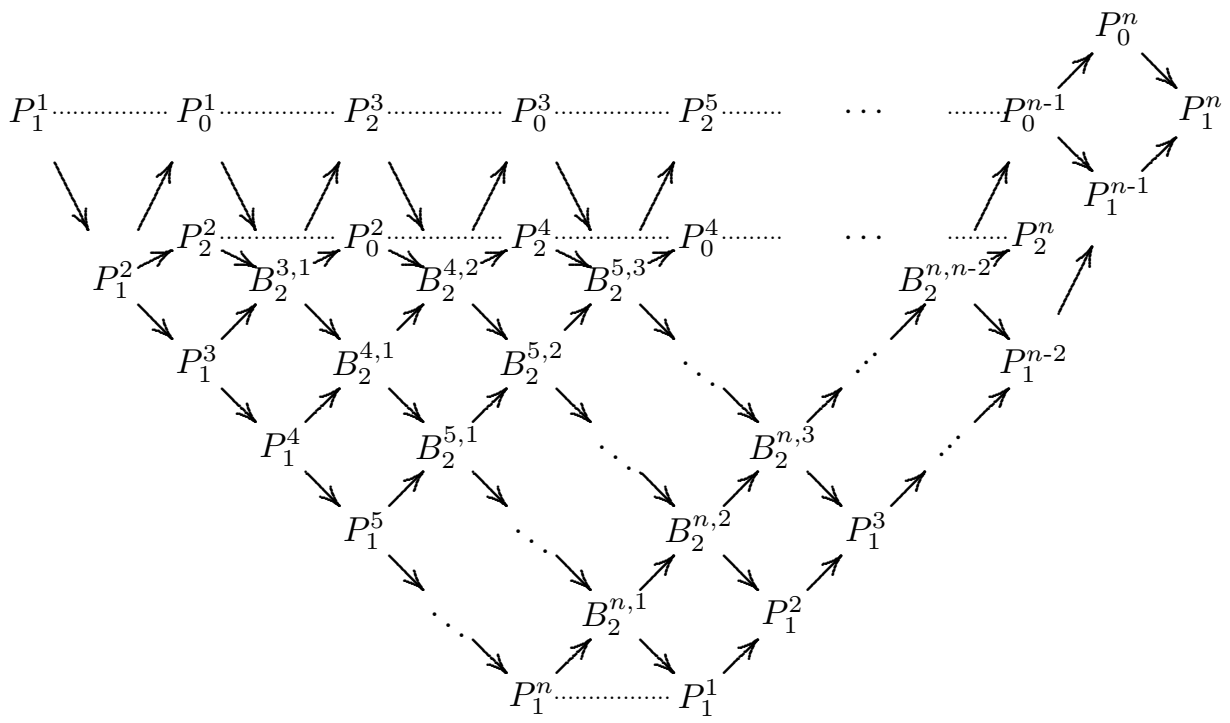


For each pair $(X, Y)$ of indecomposable objects in $\mathcal{S}_{2}(k)$ we determine in the table below the dimension of the $k$-space $\operatorname{Hom}_{\mathcal{S}}(X, Y)$. Most of the numbers are taken from [17, Lemma 4].

\begin{tabular}{|r|c|c|c|c|}
\hline \multicolumn{5}{|c|}{ Dimensions of Spaces $\operatorname{Hom}(X, Y), X, Y \in \operatorname{ind} \mathcal{S}_{2}(k)$} \\
\hline \hline$X$ & $Y=P_{0}^{m}$ & $P_{2}^{m}$ & $B_{2}^{m, r}$ & $P_{1}^{m}$ \\
\hline$P_{0}^{\ell}$ & $\min \{\ell, m\}$ & $\min \{\ell, m\}$ & $\begin{array}{c}\min \{\ell, m\} \\
+\min \{\ell, r\}\end{array}$ & $\min \{\ell, m\}$ \\
\hline$P_{2}^{\ell}$ & $\min \{\ell-2, m\}$ & $\min \{\ell, m\}$ & $\begin{array}{c}\min \{\ell-1, m\} \\
+\min \{\ell-1, r\}\end{array}$ & $\min \{\ell-1, m\}$ \\
\hline \multirow{B}{*}{$B_{2}^{\ell, t}$} & $\min \{\ell-1, m\}$ \\
$+\min \{t-1, m\}$ & $\min \{\ell, m\}$ & $\begin{array}{l}\min \{\ell-1, m\} \\
+\min \{t, m\} \\
+\min \{\ell-1, r\} \\
+\min \{t, r\} \\
-\mathbf{1}\{\ell>m \text { and } t \leq r\}\end{array}$ & $\min \{\ell-1, m\}$ \\
$+\min \{t, m\}$ & $\min \{\ell, m\}$ \\
\hline$P_{1}^{\ell}$ & $\min \{\ell-1, m\}$ & $\min \{\ell, m\}$ & $\min \{\ell-1, r\}$ & $\min \{\ell, m\}$ \\
\hline
\end{tabular}

We denote by 1 the characteristic function corresponding to the property specified in parantheses.

It will help us simplify the presentation in the next section by introducing the notation

$$
B_{2}^{m, m-1}=P_{2}^{m} \oplus P_{0}^{m-1}
$$

for $m \geq 2$. We observe that the notation is consistent with the formulae above.

4.2. How operations change the hom spaces. Throughout this section, $Y, Z \in$ $\mathcal{S}_{2}$ will be objects of the same partition type. We introduce two matrices, the multiplicity matrix $\delta M=\delta M(Y, Z)$ and the hom matrix $\delta H=\delta H(Y, Z)$; in each case the indexing set is the set of isomorphism types of indecomposable objects in $\mathcal{S}_{2}$. The matrices are defined as follows:

$$
\delta M_{X}=\mu_{X}(Z)-\mu_{X}(Y) \text { and } \delta H_{X}=[X, Z]_{\mathcal{S}}-[X, Y]_{\mathcal{S}},
$$

where $[X, Z]_{\mathcal{S}}=\operatorname{dim} \operatorname{Hom}_{\mathcal{S}}(X, Z)$ and where $\mu_{X}(Z)$ denotes the number of direct summands of $Z$ that are isomorphic to $X$.

We visualize the matrices by indicating the value at $X \in \operatorname{ind} \mathcal{S}_{2}$ in the position of $X$ in the Auslander-Reiten quiver for $\mathcal{S}_{2}^{n}$, with $n$ large enough. We sketch this quiver as follows: The modules on the top line are the $P_{2}^{m}$, and those on the second line are the $P_{0}^{r}$; the modules in the triangle have type $B_{2}^{m, r}$. The modules $P_{1}^{r}$ are repeated twice, on the diagonal at the left and on the anti-diagonal at the right.

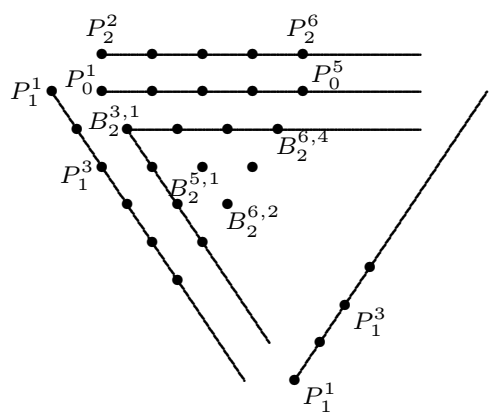


Lemma 4.2. Suppose $Y, Z \in \mathcal{S}_{2}$ have the same partition type $(\alpha, \beta, \gamma)$.

(1) The Hom matrix $\delta H(Y, Z)$ has zero entry at each position corresponding to a module $P_{1}^{1}, P_{0}^{m}, P_{2}^{m}$ where $m \in \mathbb{N}$.

(2) Along each diagonal in the Hom matrix, the entries eventually become constant:

$$
\lim _{m \rightarrow \infty} \delta H(Y, Z)_{B_{2}^{m, r}}=\delta H(Y, Z)_{P_{1}^{m}}
$$

Proof. For the first statement we use the table in the previous section to verify that the dimensions of homomorphism spaces are determined by the partition type:

$$
\begin{aligned}
{\left[P_{1}^{1}, Y\right]_{\mathcal{S}} } & =\bar{\alpha}_{1}, \\
{\left[P_{0}^{m}, Y\right]_{\mathcal{S}} } & =\bar{\beta}_{1}+\cdots+\bar{\beta}_{m}, \\
{\left[P_{2}^{m}, Y\right]_{\mathcal{S}} } & =\bar{\alpha}_{1}+\bar{\alpha}_{2}+\bar{\gamma}_{1}+\cdots \bar{\gamma}_{m-2} .
\end{aligned}
$$

For the second assertion, let $n=\beta_{1}$ be the nilpotency index of the operator acting on $Y$. By comparing the third and the fourth row in the table, we see that for each $m>n$ the equality $\left[B_{2}^{m, r}, Y\right]_{\mathcal{S}}=|\beta|+\left[P_{1}^{r}, Y\right]_{\mathcal{S}}$ holds where $|\beta|=\sum_{i} \beta_{i}$.

We determine how the matrices change for each operation on the arc diagram.

(A) Suppose $Z$ is obtained from $Y$ by a transformation

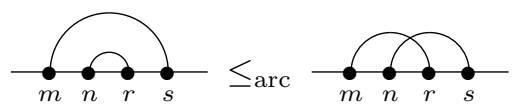

where $m>n>r>s$ and $n>r+1$. Recall that the two arcs in the diagram for $Y$ represent direct summands $B_{2}^{m, s}$ and $B_{2}^{n, r}$, which are replaced by the summands $B_{2}^{m, r}$ and $B_{2}^{n, s}$ in $Z$ that give rise to the corresponding arcs in the diagram for $Z$.

Thus, the multiplicity matrix is as follows:

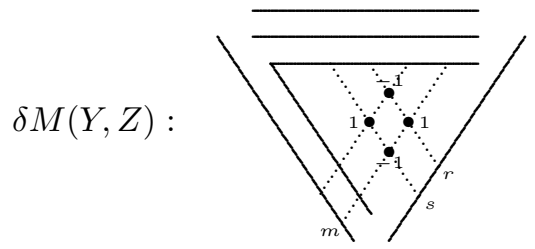

Note that the marked points correspond to a short exact sequence

$$
0 \longrightarrow B_{2}^{n, s} \longrightarrow B_{2}^{m, s} \oplus B_{2}^{n, r} \longrightarrow B_{2}^{m, r} \longrightarrow 0,
$$

which serves as a witness for the implication

$$
Y \leq_{\text {arc }} Z \Longrightarrow Y \leq_{\text {ext }} Z .
$$

Next we determine the Hom matrix $\delta H=\delta H\left(B_{2}^{m, s} \oplus B_{2}^{n, r}, B_{2}^{n, s} \oplus B_{2}^{m, r}\right)$ : By Lemma 4.2. $\delta H_{P_{0}^{\ell}}=0=\delta H_{P_{2}^{\ell}}$. We compute using the table in Section 4.1. $\delta H_{P_{1}^{\ell}}=0$ and

$$
\delta H_{B_{2}^{\ell, t}}=\mathbf{1}\{n<\ell \leq m \text { and } s<t \leq r\} .
$$

Thus, the only indecomposables $X \in \mathcal{S}_{2}$ for which $\delta H_{X} \neq 0$ are the $B_{2}^{\ell, t}$ where $n<\ell \leq m$ and $s<t \leq r$. For each such module $X$ we have $\delta H_{X}=1$. They lie in 
the shaded region in the diagram below:

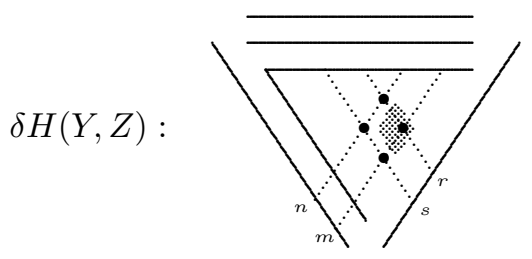

(A') In this case $Z$ is obtained from $Y$ by an arc operation of type

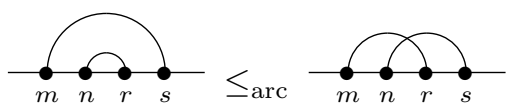

where $m>n>r>s$, as in (A), but now $n=r+1$. Here the two arcs in the diagram for $Y$ represent direct summands $B_{2}^{m, s}$ and (corresponding to the arc from $n$ to $r) P_{2}^{n}$ and $P_{0}^{r}$; they are replaced by the summands $B_{2}^{m, r}$ and $B_{2}^{n, s}$ in $Z$.

In this case, the short exact sequence demonstrates the implication $Y \leq_{\text {arc }} Z \Rightarrow$ $Y \leq{ }_{\text {ext }} Z$ :

$$
0 \longrightarrow B_{2}^{n, s} \longrightarrow B_{2}^{m, s} \oplus P_{2}^{n} \oplus P_{0}^{r} \longrightarrow B_{2}^{m, r} \longrightarrow 0 .
$$

We picture the corresponding multiplicity and Hom matrices. In view of the observation after the table in Section 4.1 the computations for the Hom matrix in (A) are still valid in this case: $\delta H_{X}=1$ for $X=B_{2}^{\ell, t}$, where $n<\ell \leq m, s<t \leq r$.

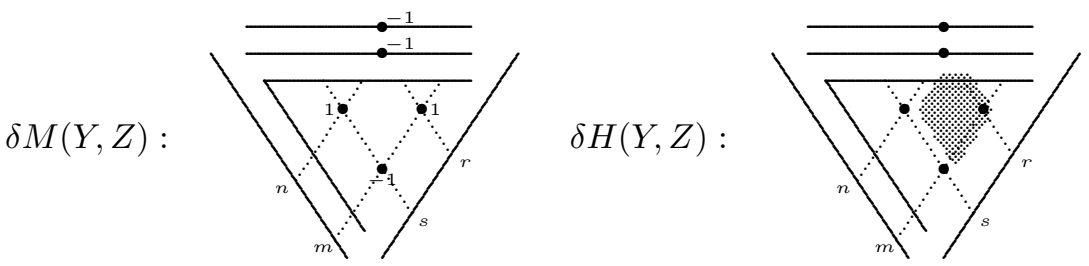

(B) Next we deal with the transformation

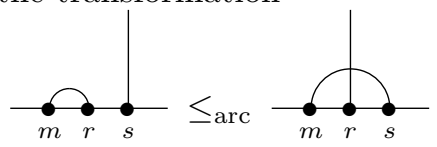

where $m>r>s$. We consider the two cases where $m-1>r$ and where $m-1=r$ simultaneously using the notation $B_{2}^{m, m-1}=P_{2}^{m} \oplus P_{0}^{m-1}$. Thus, $Y$ has summands $B_{2}^{m, r}$ and $P_{1}^{s}$ which are replaced in the transformation by summands $B_{2}^{m, s}$ and $P_{1}^{r}$ for $Z$.

The following short exact sequence demonstrates that $Y \leq_{\text {arc }} Z$ implies $Y \leq_{\text {ext }}$ $Z$ :

$$
0 \longrightarrow B_{2}^{m, s} \longrightarrow B_{2}^{m, r} \oplus P_{1}^{s} \longrightarrow P_{1}^{r} \longrightarrow 0 .
$$

Here are the corresponding multiplicity and Hom-matrices:

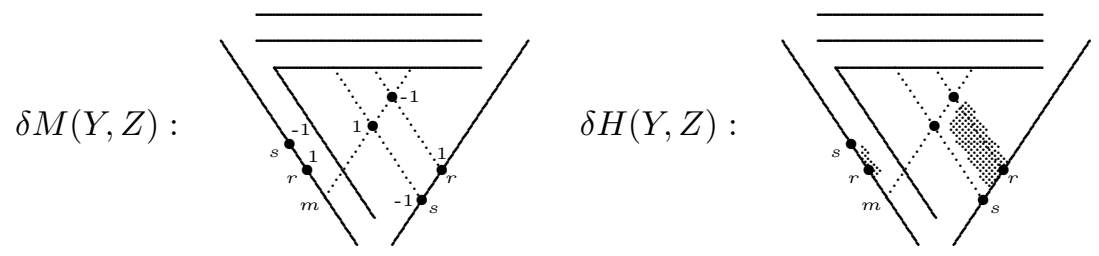


Here $\delta H_{X}=1$ for modules $X$ of type $B_{2}^{\ell, t}$ where $m<\ell$ and $s<t \leq r$, and for modules of type $P_{1}^{t}$ where $s<t \leq r$.

(C) Here $Z$ is obtained from $Y$ by a transformation of type

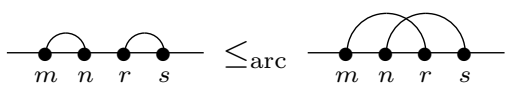

where $m>n>r>s$. Recall that the two arcs in the diagram for $Y$ represent direct summands $B_{2}^{m, n}$ and $B_{2}^{r, s}$, which are replaced by the summands $B_{2}^{m, r}$ and $B_{2}^{n, s}$ in $Z$ that give rise to the corresponding arcs in the diagram for $Z$. Note that we are really dealing with 4 cases depending on whether $m=n+1$ (in which case $B_{2}^{m, n}=P_{2}^{m} \oplus P_{0}^{n}$ is decomposable) or $m>n+1$, and whether $r=s+1$ (in which case $B_{2}^{r, s}=P_{2}^{r} \oplus P_{0}^{s}$ is decomposable) or $r>s+1$. We picture the matrices only for the case where $m>n+1$ and $r>s+1$; the modifications for the remaining cases are as in (A) and $\left(\mathbf{A}^{\prime}\right)$.

The multiplicity matrix is as follows:

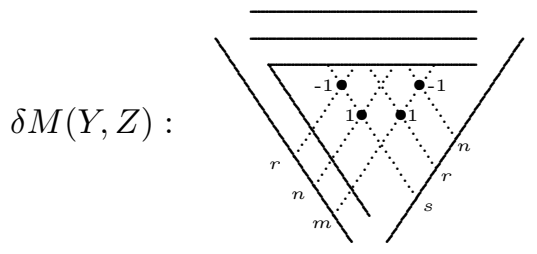

The marked points correspond to a short exact sequence

$$
0 \longrightarrow B_{2}^{m, r} \longrightarrow B_{2}^{m, n} \oplus B_{2}^{r, s} \longrightarrow B_{2}^{n, s} \longrightarrow 0,
$$

which confirms the implication $Y \leq_{\text {arc }} Z \Rightarrow Y \leq_{\text {ext }} Z$. For the Hom matrix $\delta H=$ $\delta H\left(B_{2}^{m, n} \oplus B_{2}^{r, s}, B_{2}^{n, s} \oplus B_{2}^{m, r}\right)$ we use the table in Section 4.1.

$$
\begin{aligned}
\delta H_{P_{1}^{\ell}} & =\mathbf{1}\{r<\ell \leq n\}, \\
\delta H_{B_{2}^{\ell, t}} & =\mathbf{1}\{r<\ell \leq n \text { and } t \leq s\}+\mathbf{1}\{m<\ell \text { and } r<t \leq n\} .
\end{aligned}
$$

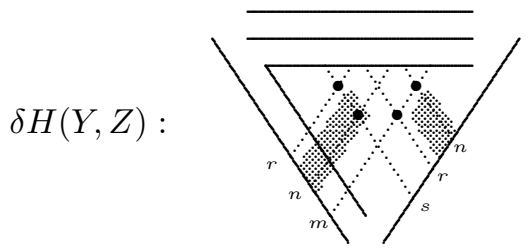

(D) Finally, we deal with the transformation

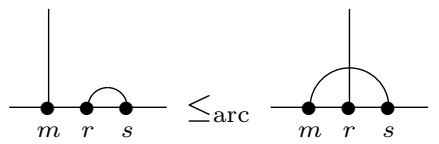

where $m>r>s$. Thus, $Y$ has summands $B_{2}^{r, s}$ and $P_{1}^{m}$ which are replaced in the transformation by summands $B_{2}^{m, s}$ and $P_{1}^{r}$ for $Z$. The following short exact sequence demonstrates that $Y \leq_{\text {arc }} Z$ implies $Y \leq_{\text {ext }} Z$ :

$$
0 \longrightarrow P_{1}^{r} \longrightarrow P_{1}^{m} \oplus B_{2}^{r, s} \longrightarrow B_{2}^{m, s} \longrightarrow 0 .
$$


Here are the corresponding multiplicity and Hom-matrices:

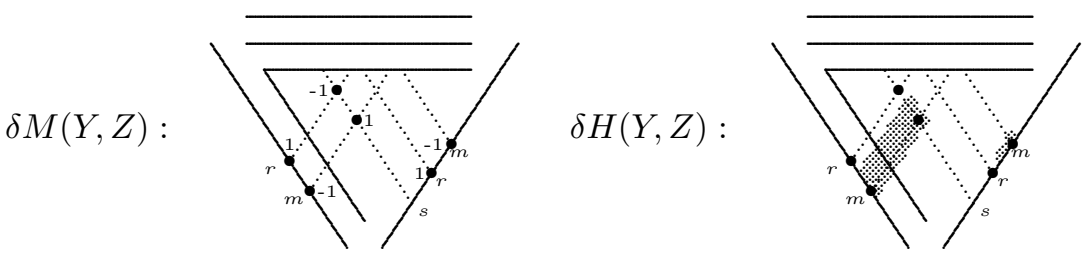

We proved the following fact.

Lemma 4.3. Let $k$ be an arbitrary field and let $Y, Z \in \mathcal{S}_{2}(k)$ have the same partition type $(\alpha, \beta, \gamma)$. If $Y \leq_{\operatorname{arc}} Z$, then $Y \leq_{\mathrm{ext}} Z$.

Note that the Hom matrix determines the multiplicity matrix uniquely. Namely, let $A$ be a noninjective indecomposable object with Auslander-Reiten sequence $0 \rightarrow A \rightarrow \bigoplus B_{i} \rightarrow C \rightarrow 0$ in $\mathcal{S}_{2}^{n}$. Then the multiplicity of $A$ as a direct summand of $Y$ is given by the contravariant defect $\mu_{A}(Y)=[A, Y]+[C, Y]-\sum_{i}\left[B_{i}, Y\right]$.

The following consequence, which is technical but easy to show, will be used in the proof of Proposition 4.5 .

Lemma 4.4. Suppose that both $Y, Z \in \mathcal{S}_{2}^{n}$ have partition type $(\alpha, \beta, \gamma)$ with $\alpha_{1} \leq 2$.

(1) The nonzero part of the Hom matrix $\delta H(Y, Z)$ is contained in the union of the $\tau$-orbits for $X=B_{2}^{3,1}, \ldots, B_{2}^{n, 1}$; they form a Moebius band, i.e. a quiver of type $\mathbb{Z}_{n-2}$ with suitable identifications.

(2) For each noninjective $A \in$ ind $\mathcal{S}_{2}^{n}$, the entry $\delta M_{A}=\delta M(Y, Z)_{A}$ in the multiplicity matrix can be read from the the restriction of the Hom matrix to the Moebius band by a formula of type

$$
\delta M_{A}=\beta_{A}+\beta_{C}-\sum_{i} \beta_{B_{i}},
$$

where $0 \rightarrow A \rightarrow \bigoplus_{i} B_{i} \rightarrow C \rightarrow 0$ is the Auslander-Reiten sequence starting at $A$ and

$$
\beta_{X}= \begin{cases}\delta H(Y, Z)_{X} & \text { if } X \text { is in the Moebius band } \\ 0 & \text { otherwise. }\end{cases}
$$

Proof. The first statement follows from Lemma 4.2 and the second from the contravariant defect formula above.

4.3. An example. In this section we present the example from the introduction to Chapter 4 in detail. The arc diagrams

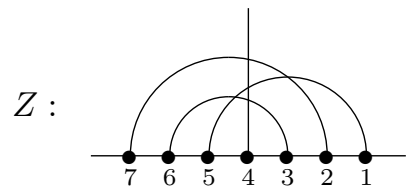

$Y:$

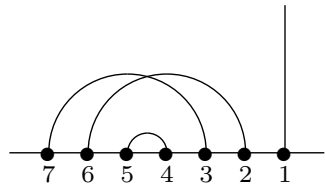

represent the modules $Y=B_{2}^{7,3} \oplus B_{2}^{6,2} \oplus P_{2}^{5} \oplus P_{0}^{4} \oplus P_{1}^{1}$ and $Z=B_{2}^{7,2} \oplus B_{2}^{6,3} \oplus$ $B_{2}^{5,1} \oplus P_{1}^{4}$.

We first compute the Hom-matrix to verify that $Y \leq_{\text {hom }} Z$. In this matrix, the entries on the first diagonal represent the numbers $\delta H_{B_{2}^{3,1}}, \delta H_{B_{2}^{4,1}}, \ldots$ By 
Lemma 4.2, there are also zeros along the two top rows, which we indicate by solid lines:

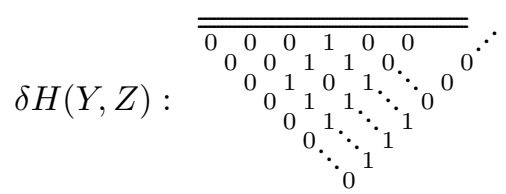

We ask: Is it true that $Y \leq \operatorname{arc} Z$ ? If so, which operations transform the arc diagram of $Z$ into the arc diagram of $Y$ ?

First we consider again the single operation of type ( $\left.\mathbf{B}^{\prime}\right)$ on $Z$, replacing the arc from 5 to 1 , i.e. the bipicket $B_{2}^{5,1}$ and the pole at 4 , i.e. the picket $P_{1}^{4}$ by the arc given by the pickets $P_{2}^{5}, P_{0}^{4}$ and the pole at 1 given by the picket $P_{1}^{1}$, to obtain the module $\tilde{Z}$ with the following arc diagram:

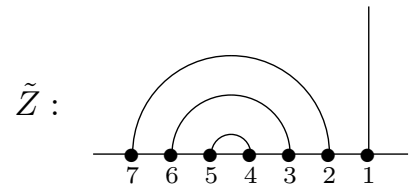

This single misstep yields $\tilde{Z} \leq_{\text {hom }} Y$ since, as we have seen in Section 4.2. part (B'), all entries in the big parallelogram in the Hom-matrix have been reduced by 1.

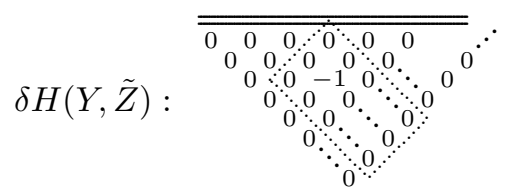

So we start over. Now we focus on the smaller parallelogram in the Hom-matrix, as indicated.

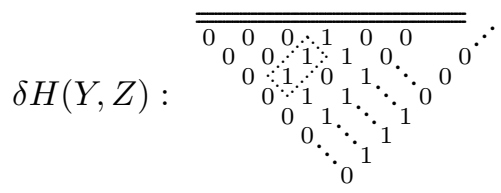

There are two reasons for considering this parallelogram: (1) On the left of the left corner of the parallelogram, and in the right corner, there are positive entries in the multiplicity diagram; see Section 4.2. Those entries indicate the arcs and poles involved in the operation which produces $Z^{\prime}$ from $Z$.

(2) Unlike the big parallelogram, there are only positive entries in the smaller parallelogram. This will make sure that $Y \leq_{\text {hom }} Z^{\prime}$ holds.

The corresponding operation of type $(\mathbf{A})$ replaces the bipickets $B_{2}^{5,1}$ and $B_{2}^{6,3}$ in $Z$ (representing arcs from 5 to 1 and from 6 to 3 , respectively) by bipickets $B_{2}^{6,1}$ and $B_{2}^{5,3}$ in $Z^{\prime}$, so $Z^{\prime}$ is given by the following arc diagram. We also indicate the new Hom-matrix.

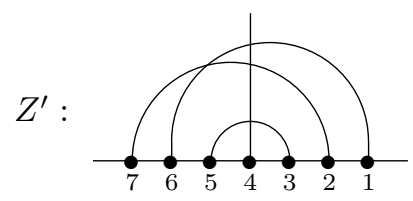

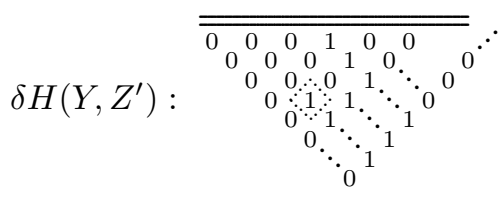


We pick a new parallelogram satisfying conditions (1) and (2) (alternatively, we could have chosen the parallelogram given by the upper diagonal in the region marked by the 1's) and perform the operation indicated: Replace in $Z^{\prime}$ the bipickets $B_{2}^{6,1}$ and $B_{2}^{7,2}$ by bipickets $B_{2}^{6,2}$ and $B_{2}^{7,1}$ in $Z^{\prime \prime}$. The arc diagram and Hom-matrix are as follows:

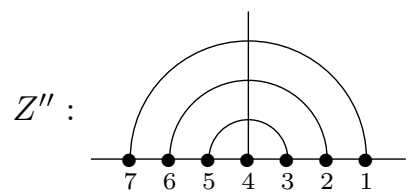

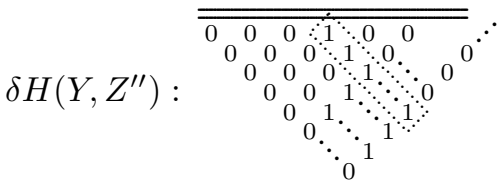

The parallelogram suggests an operation of type (B'), namely to replace the bipicket $B_{2}^{5,3}$ and the picket $P_{1}^{4}$ in $Z^{\prime \prime}$ by pickets $P_{2}^{5}, P_{0}^{4}$ and $P_{1}^{3}$ in $Z^{\prime \prime \prime}$ (note that the first two pickets represent an arc from 5 to 4 , the last is a pole at 3 ).

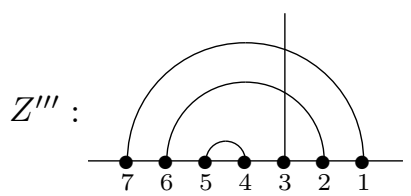

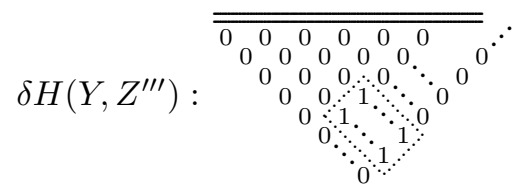

Finally, an operation of type (B) reduces the Hom-matrix to zero and yields the module $Y$ : We replace the bipicket $B_{2}^{7,1}$ and the picket $P_{1}^{3}$ in $Z^{\prime \prime \prime}$ by the bipicket $B_{2}^{7,3}$ and the picket $P_{1}^{1}$ for $Y$. Done!

$$
Y \leq_{\text {arc }} Z^{\prime \prime \prime} \leq_{\text {arc }} Z^{\prime \prime} \leq_{\text {arc }} Z^{\prime} \leq_{\text {arc }} Z \text {. }
$$

4.4. Operations on the arc diagram. In this section we complete the proof of Theorem 4.1. Throughout we assume that $Y, Z \in \mathcal{S}_{2}(k)$ have the same partition type $(\alpha, \beta, \gamma)$. To prove the implication $Y \leq_{\text {hom }} Z \Rightarrow Y \leq_{\text {arc }} Z$, we assume that $Y \leq_{\text {hom }} Z$ holds and apply the following result repeatedly.

Proposition 4.5. Suppose $Y$ and $Z$ have the same partition type, $Y \leq_{\text {hom }} Z$ and $Y \neq Z$. Then there is an operation on the arc diagram for $Z$ of type $(\mathbf{A}),(\mathbf{B})$, (C) or (D) which yields a module $Z^{\prime}$ such that

$$
Z^{\prime} \leq_{\text {arc }} Z, \quad Z^{\prime} \neq Z, \quad \text { and } \quad Y \leq_{\text {hom }} Z^{\prime} .
$$

In the proof we will use the following result.

Lemma 4.6. Consider the following matrix of integers:

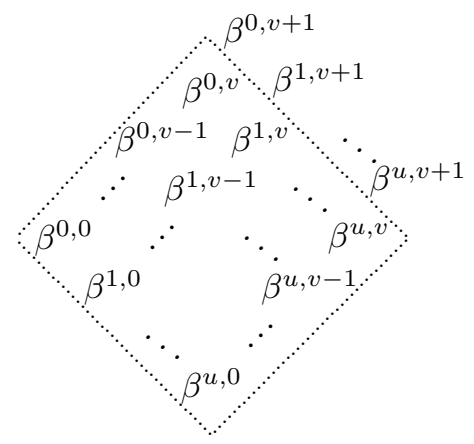


Suppose the following conditions are satisfied:

(1) All entries are nonnegative.

(2) The numbers $\beta^{0,0}, \beta^{0,1}, \ldots, \beta^{0, v}$ are strictly positive.

(3) $\beta^{0, v+1}=0$.

(4) For each $0 \leq i \leq u-1,0 \leq j \leq v$,

$$
\delta^{i j}=\beta^{i, j}+\beta^{i+1, j+1}-\beta^{i, j+1}-\beta^{i+1, j} \leq 0 .
$$

Then all entries in the parallelogram are positive: $\beta^{i, j}>0$ for each $0 \leq i \leq u$, $0 \leq j \leq v$.

Proof. By assumption, $\beta^{0, v}, \beta^{0, v-1}, \ldots, \beta^{0,0}$ are all strictly positive. Note that

$$
\beta^{1, v}=\beta^{0, v}+\beta^{1, v+1}-\beta^{0, v+1}-\delta^{0, v} \geq \beta^{0, v}>0 .
$$

Since $\delta^{0, j}+\cdots+\delta^{0, v}=\beta^{0, j}+\beta^{1, v+1}-\beta^{0, v+1}-\beta^{1, j}$, it follows that for each $0 \leq j \leq v$,

$$
\beta^{1, j}=\beta^{0, j}+\beta^{1, v+1}-\beta^{0, v+1}-\left(\delta^{0, j}+\cdots+\delta^{0, v}\right) \geq \beta^{0, j}>0 .
$$

In general, since for each $1 \leq i \leq u, 0 \leq j \leq v$,

$$
\delta(i, j)=\sum_{0 \leq h<i, j \leq \ell \leq v} \delta^{h, \ell}=\beta^{0, j}+\beta^{i, v+1}-\beta^{i, j}-\beta^{0, v+1} \leq 0,
$$

we have

$$
\beta^{i, j}=\beta^{0, j}+\beta^{i, v+1}-\beta^{0, v+1}-\delta(i, j) \geq \beta^{0, j}>0 .
$$

Also the dual version holds:

Lemma 4.7. Consider the following matrix of integers:

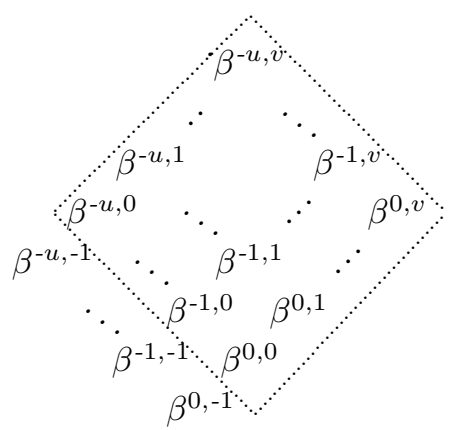

Suppose that in addition to conditions (1) and (2) from the previous lemma the following are also satisfied:

(3') $\beta^{0,-1}=0$.

(4') For each $-u \leq i<0,-1 \leq j<v$,

$$
\delta^{i j}=\beta^{i, j}+\beta^{i+1, j+1}-\beta^{i, j+1}-\beta^{i+1, j} \leq 0 .
$$

Then all entries in the parallelogram are positive: $\beta^{i, j}>0$ for each $-u \leq i \leq 0$, $0 \leq j \leq v$.

Proof of Proposition 4.5. The set-up. We assume that the entries in the Hommatrix $\delta H(Y, Z)$ are all nonnegative and that at least one entry is positive. 
The goal. We show that there is a parallelogram in the Hom-matrix (in the shape of one of the shaded regions in Section 4.2) which satisfies the following two conditions:

(P1) All entries within the parallelogram are strictly positive.

(P2) The two indecomposable modules $X^{\prime}$ and $X^{\prime \prime}$ corresponding to the right corner of the parallelogram and to the point just left of the left corner, respectively, occur with higher multiplicity as direct summands of $Z$ than as a direct summand of $Y$.

For illustration, we repeat the situation before the first move in the example. The parallelogram is indicated.

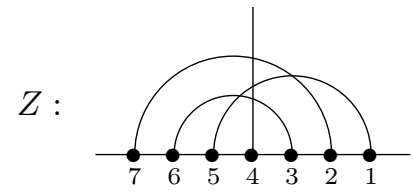

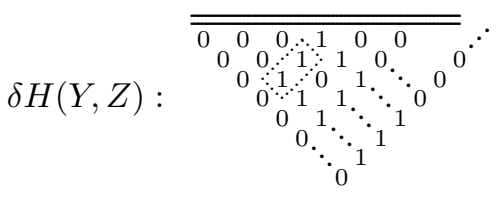

Step 1 . We choose a number $n>\beta_{1}$ and work in the category $\mathcal{S}_{2}^{n}$. Recall that in the Hom matrix, the nonzero entries are confined to the orbits given by the modules $B_{2}^{3,1}, \ldots, B_{2}^{n, 1}$ which form a stripe of type $\mathbb{Z} \mathbb{A}_{n-2}$, with identifications. For the purpose of this algorithm, we view the Hom matrix as a stripe of type $\mathbb{Z} \mathbb{A}_{n}$, with the orbits of the $B_{2}^{3,1}, \ldots, B_{2}^{n, 1}$ in the center and two orbits of zeros on the boundary.

Pick a sequence $\beta^{0,0}, \ldots, \beta^{0, v}$ of positive entries arranged along an anti-diagonal in the Hom matrix, such that the neighboring entries on the anti-diagonal, $\beta^{0,-1}$ and $\beta^{0, v+1}$, are both zero. This is possible since the entries in the matrix are nonnegative, since there is at least one positive entry and since there are zeros on the boundary orbits.

In the example, we have picked the anti-diagonal $\beta^{0,0}=\delta H_{B_{2}^{6,2}}=1, \beta^{0,1}=$ $\delta H_{B_{2}^{6,3}}=1, \beta^{0,2}=\delta H_{B_{2}^{6,4}}=1$.

Step 2. We determine the right corner of the parallelogram. Put $u=0$.

Consider the sequence $\delta^{u, 0}, \ldots, \delta^{u, v}$ of entries in the multiplicity matrix in the positions given by the $\beta^{u, 0}, \ldots, \beta^{u, v}$ in the Hom matrix.

If one of the entries in the sequence $\delta^{u, 0}, \ldots, \delta^{u, v}$ is positive, put $u^{\prime \prime}=u$ and let $\delta^{u^{\prime \prime}, w^{\prime \prime}}$ be the first such entry. In this case, $\left(u^{\prime \prime}, w^{\prime \prime}\right)$ will be the right corner of the rectangle and $X^{\prime \prime}$, the object corresponding to the position $\left(u^{\prime \prime}, w^{\prime \prime}\right)$, will be a summand occurring with higher multiplicity in $Z$ than in $Y$. We are done with the second step.

By Lemma 4.6. we obtain that all the numbers $\beta^{u+1,0}, \ldots, \beta^{u+1, v}$ in the Hom matrix, on the anti-diagonal just under the previous anti-diagonal, are positive. Put $u:=u+1$ and proceed with the paragraph under Step 2.

Note that this process terminates: For $u$ large enough, $\beta^{u, 0}$ will correspond to a point on the boundary of the Hom matrix. Hence $\beta^{u, 0}=0$.

In the example, the number $\delta^{0,2}=\delta M_{B_{2}^{6,3}}=\beta^{0,2}+\beta^{1,3}-\beta^{0,3}-\beta^{1,2}=1$ is the first positive value among the entries in the multiplicity matrix on the anti-diagonal. Thus, $X^{\prime \prime}=B_{2}^{6,3}$.

Step 3. We determine the left corner of the parallelogram. Put $u=0$ and $v=v^{\prime \prime}$.

Consider the sequence $\delta^{u-1,-1}, \ldots, \delta^{u-1, v-1}$ of entries in the multiplicity matrix in the positions just left of the $\beta^{u, 0}, \ldots, \beta^{u, v}$ in the Hom matrix. 
If one of the entries in the sequence $\delta^{u-1,-1}, \ldots, \delta^{u-1, v-1}$ is positive, put $u^{\prime}=$ $u-1$ and let $\delta^{u^{\prime}, w^{\prime}}$ be the last such entry. In this case, $\left(u^{\prime}, w^{\prime}\right)$ will be the point just left of the left corner of the rectangle and $X^{\prime}$, the object corresponding to the position $\left(u^{\prime}, w^{\prime}\right)$, will be a summand occurring with higher multiplicity in $Z$ than in $Y$. We are done with the third step.

If none of the entries in $\delta^{u-1,-1}, \ldots, \delta^{u-1, v-1}$ is positive, then by Lemma 4.7. all the numbers $\beta^{u-1,0}, \ldots, \beta^{u-1, v}$ in the Hom matrix, on the anti-diagonal just above the previous anti-diagonal, are positive. Put $u:=u-1$ and proceed with the paragraph under Step 3.

Note that this process terminates: For $-u$ large enough, the position corresponding to $\beta^{u, 0}$ will be a point on the boundary of the Hom matrix, so $\beta^{u, 0}=0$.

In the example, $\delta^{-1,-1}=\delta M_{B_{2}^{5,1}}=1$ is the last (and first) positive entry in the multiplicity matrix on the line above the original anti-diagonal. Thus, $X^{\prime}=B_{2}^{5,1}$.

Conclusion. In each case the parallelogram marked off by $X^{\prime}$ and $X^{\prime \prime}$ is of one of the types (A), (B), (C) or (D) in Section 4.2. (Namely, if none of the modules $P_{1}^{r}$ is in the rectangle, then the type is (A); if $X^{\prime \prime}$ is one of the $P_{1}^{r}$, then the type is (B); in case $X^{\prime} \cong P_{1}^{r}$ for some $r$, then the type is (D); and finally, if there are modules $P_{1}^{r}$ in the rectangle, but neither $X^{\prime}$ nor $X^{\prime \prime}$ has this form, then the type is (C).) Write $Z=Z_{0} \oplus X^{\prime} \oplus X^{\prime \prime}$ and let $X_{1}, \ldots, X_{s}(s=2$ or 3$)$ be the modules corresponding to the entry -1 in the multiplicity matrix for the arc operation. Put $Z^{\prime}=Z_{0} \oplus X_{1} \oplus \cdots \oplus X_{s}$. By this construction, $Z^{\prime} \leq_{\text {arc }} Z$. Moreover,

$$
\delta H\left(Y, Z^{\prime}\right)_{X}=\delta H(Y, Z)_{X}+ \begin{cases}-1 & \text { if } X \text { is in the parallelogram, } \\ 0 & \text { otherwise. }\end{cases}
$$

Since the entries for $\delta H(Y, Z)$ within the parallelogram are all positive, the matrix $\delta H\left(Y, Z^{\prime}\right)$ is nonnegative, and hence

$$
Y \leq_{\text {hom }} Z^{\prime} \quad \text { and } \quad Z^{\prime} \leq_{\text {arc }} Z .
$$

Proof of Theorem 4.1. Assume that $Y \leq_{\text {hom }} Z$. Since each application of Proposition 4.5 reduces the number of crossings in the arc diagram, a finite number of steps suffice to produce a sequence of modules $Z, Z^{\prime}, Z^{\prime \prime}, \ldots, Z^{(m)}=Y$ such that

$$
Y=Z^{(m)} \leq_{\text {arc }} Z^{(m-1)} \leq_{\text {arc }} \cdots \leq_{\text {arc }} Z^{\prime \prime} \leq_{\text {arc }} Z^{\prime} \leq_{\text {arc }} Z .
$$

\section{Dimensions via Hall polynomials}

In this section we investigate the set $V_{\alpha, \gamma}^{\beta}$. We recall that if $k$ is an algebraically closed field,

$$
V_{\alpha, \gamma}^{\beta}(k)=\bigcup_{\Gamma} V_{\Gamma}(k)=\bigcup_{\Gamma} \bigcup_{\Pi} V_{\Pi}(k),
$$

where the first union is indexed by all LR-tableaux $\Gamma$ of type $(\alpha, \beta, \gamma)$ and the second union is indexed by all Klein tableaux $\Pi$ refining $\Gamma$, and where $V_{\Pi}(k)=G_{f}$ is the orbit of a point $\left(N_{\alpha}(k), N_{\beta}(k), f\right) \in V_{\alpha, \gamma}^{\beta}(k)$ with Klein tableau $\Pi$.

Correspondingly, if $k$ is a finite field of $q$ elements, there is the following sum formula for Hall polynomials:

$$
h_{\alpha, \gamma}^{\beta}(q)=\sum_{\Gamma} h_{\Gamma}(q)=\sum_{\Gamma} \sum_{\Pi} h_{\Pi}(q),
$$


where the indices are as above. The polynomials $h_{\Gamma}$ are monic of the same degree $n(\beta)-n(\alpha)-n(\gamma)$, while the polynomials $h_{\Pi}$ are monic of degree $n(\beta)-n(\alpha)-$ $n(\gamma)-x(\Pi)$ where $x(\Pi)$ denotes the deviation from dominance [8, Corollaries 1-3].

5.1. $\mathbb{Z}$-forms of objects. The following lemma shows that the categories $\mathcal{N}(k)$ and $\mathcal{S}_{2}(k)$ can be defined over $\mathbb{Z}$ (independently on the field $k$; see [5], 6]). Denote by $\mathcal{N}(\mathbb{Z})$ the category of all systems $(V, \varphi)$, where $V$ is a finitely generated free abelian group and $\varphi: V \rightarrow V$ is a nilpotent $\mathbb{Z}$-homomorphism. The morphisms are defined in the usual way. For any field $k$, let $(V, \varphi) \otimes k=\left(V \otimes_{\mathbb{Z}} k, \varphi \otimes_{\mathbb{Z}}\right.$ id $)$. Similarly, denote by $\mathcal{S}(\mathbb{Z})$ the category of all systems $(V, W, \psi)$, where $V, W$ are objects in $\mathcal{N}(\mathbb{Z})$ and $\psi: V \rightarrow W$ is a morphism in $\mathcal{N}(\mathbb{Z})$. Morphisms in $\mathcal{S}(\mathbb{Z})$ and the functor

$$
-\otimes k: \quad \mathcal{S}(\mathbb{Z}) \rightarrow \mathcal{S}(k)
$$

are defined analogously.

Lemma 5.1. (1) For any partition $\alpha$ there exists an object $N_{\alpha}(\mathbb{Z})=(V, \varphi)$ in $\mathcal{N}(\mathbb{Z})$, such that for any field $k$ :

$$
N_{\alpha}(\mathbb{Z}) \otimes k \cong N_{\alpha}(k) .
$$

Moreover there exists a $\mathbb{Z}$-basis of $V$ such that the matrix of $\varphi$ in this basis has coefficients equal to 0 or 1.

(2) For any Klein tableau $\Pi$ with entries at most two there exists an object $M_{\Pi}(\mathbb{Z})=(V, W, \psi)$, in $\mathcal{S}(\mathbb{Z})$, such that for any field $k$ :

$$
M_{\Pi}(\mathbb{Z}) \otimes k \cong M_{\Pi}(k) .
$$

Moreover there exist $\mathbb{Z}$-bases of $V$ and $W$ such that the matrix of $\psi$ in these bases has coefficients equal to 0 or 1 .

Proof. (1) Let $\alpha=\left(\alpha_{1} \geq \ldots \geq \alpha_{n}\right)$ be a partition. Set $N_{\alpha}(\mathbb{Z})=(V, \varphi)$, where $V=\mathbb{Z}^{\alpha_{1}} \oplus \ldots \oplus \mathbb{Z}^{\alpha_{n}}$, and $\varphi$ is a direct sum of nilpotent Jordan blocks of sizes $\alpha_{1}, \ldots, \alpha_{n}$. It is clear that $N_{\alpha}(\mathbb{Z})$ satisfies the required conditions.

(2) Let $\Pi$ be a Klein tableau with entries at most two and let $k$ be an arbitrary field. By Theorem 2.4 the module $M_{\Pi}(k)$ is a direct sum of pickets $P_{\ell}^{m}(k)$ and bipickets $B_{2}^{m, r}(k)$. We set

$$
P_{\ell}^{m}(\mathbb{Z})=\left(N_{(\ell)}(\mathbb{Z}), N_{(m)}(\mathbb{Z}), \iota\right)
$$

and

$$
B_{2}^{m, r}(\mathbb{Z})=\left(N_{(2)}(\mathbb{Z}), N_{(m, r)}(\mathbb{Z}), \delta\right) .
$$

Note that there exist bases such that the matrices of $\iota$ and $\delta$ have entries equal to 0 and 1. Moreover $P_{\ell}^{m}(\mathbb{Z}) \otimes k \cong P_{\ell}^{m}(k)$ and $B_{2}^{m, r}(\mathbb{Z}) \otimes k \cong B_{2}^{m, r}(k)$. This finishes the proof.

5.2. Dimensions of orbits. Now let $k$ be an algebraically closed field, $\alpha, \beta, \gamma$ partitions with $\alpha_{1} \leq 2$, let $\Gamma$ be an LR-tableau of type $(\alpha, \beta, \gamma)$, $\Pi$ a Klein tableau refining $\Gamma$, and $M_{\Pi}(k)=\left(N_{\alpha}, N_{\beta}, f\right)$ an object in $\mathcal{S}_{2}(k)$ corresponding to $\Pi$. In Section 3.1 we described an algebraic group action under which $V_{\Pi}(k)=G_{f}$ is an orbit. Then we have

$$
\operatorname{dim} V_{\Pi}(k)=\operatorname{dim} \operatorname{Aut}_{\mathcal{N}}\left(N_{\alpha}(k)\right)+\operatorname{dim} \operatorname{Aut}_{\mathcal{N}}\left(N_{\beta}(k)\right)-\operatorname{dim} \operatorname{Aut}_{\mathcal{S}}\left(M_{\Pi}(k)\right),
$$


where dim is the variety dimension. The subset $V_{\Pi}(k)$ of $V_{\alpha}^{\beta}(k)$ is locally closed, because it is an orbit of an action of an algebraic group. Therefore $V_{\Pi}(k)$ is locally closed in $H_{\alpha}^{\beta}(k)$.

Recall the result of Lang and Weil [11] (see also [10, Proposition 5.6]). Let $\mathbb{F}_{p}$ be a field with $p$ elements, let $k=\overline{\mathbb{F}_{p}}$ be its algebraic closure and let $n \in \mathbb{N}$. Moreover, let $\mathcal{U} \subseteq k^{n}$ be a locally closed subset which is closed under the Frobenius automorphism $\sigma$ of $k^{n}$ (i.e. under the morphism $\sigma: k^{n} \rightarrow k^{n}$ defined by $\sigma(x)=$ $\left.\sigma\left(x_{1}, \ldots, x_{n}\right)=\left(x_{1}^{p}, \ldots, x_{n}^{p}\right)=x^{(p)}\right)$. For any finite subfield $\mathbb{F}_{q}$ of $k$, set

$$
\mathcal{U}\left(\mathbb{F}_{q}\right)=\mathcal{U} \cap \mathbb{F}_{q}^{n} .
$$

Proposition 5.3 ([11, [10]). If $\mathcal{U}$ is closed under $\sigma$, then

$$
\# \mathcal{U}\left(\mathbb{F}_{q}\right) \approx c q^{\operatorname{dim} \mathcal{U}},
$$

where $c$ is the number of irreducible components of $\mathcal{U}$ of maximal dimension, and $f(q) \approx g(q)$ if $\lim _{q \rightarrow \infty} \frac{f(q)-g(q)}{f(q)}=0$.

We use this result to determine the dimension of the orbit $V_{\Pi}(k)$ as the degree of the Hall polynomial $g_{\Pi}(t)$. Let

$$
\begin{aligned}
a_{\alpha}(q) & =\# \operatorname{Aut}_{\mathcal{N}\left(\mathbb{F}_{q}\right)}\left(N_{\alpha}\left(\mathbb{F}_{q}\right)\right), \\
a_{\Pi}(q) & =\operatorname{Aut}_{\mathcal{S}\left(\mathbb{F}_{q}\right)}\left(M_{\Pi}\left(\mathbb{F}_{q}\right)\right), \text { and } \\
g_{\Pi}(q) & =\frac{a_{\beta}(q) \cdot a_{\alpha}(q)}{a_{\Pi}(q)} .
\end{aligned}
$$

Note that

$$
\# V_{\Pi}\left(\mathbb{F}_{q}\right)=g_{\Pi}(q)
$$

and

$$
g_{\Pi}(q)=h_{\Pi}(q) \cdot a_{\alpha}(q)
$$

for any $q$ (compare [17, 3.1]).

Lemma 5.4. The function $a_{\alpha}(q)$ is a polynomial in $q$ with integral coefficients. If $\Pi$ is a Klein tableau of type $(\alpha, \beta, \gamma)$, then $a_{\Pi}(q)$ and $g_{\Pi}(q)$ are polynomials in $q$ with integral coefficients.

Proof. By [12, II.1.6] and [17, Lemma 4], $a_{\alpha}(q), a_{\Pi}(q)$ are polynomials in $q$ with integral coefficients. To prove that $g_{\Pi}(q)$ is a polynomial in $q$ with integral coefficients one can develop arguments given in [14, Section 2, Lemma (4)].

Proposition 5.5. Let $\alpha, \beta$ be partitions, let $p$ be a prime number and let $k=\overline{\mathbb{F}_{p}}$. Then

(1) $\operatorname{dim} \operatorname{Aut}_{\mathcal{N}}\left(N_{\alpha}(k)\right)=\operatorname{deg} a_{\alpha}$.

If in addition $M_{\Pi}(k)=\left(N_{\alpha}(k), N_{\beta}(k), f\right)$ is an object in $\mathcal{S}_{2}(k)$ with Klein tableau $\Pi$, then

(2) $\operatorname{dim} \operatorname{Aut}_{\mathcal{S}}\left(M_{\Pi}(k)\right)=\operatorname{deg} a_{\Pi}$,

(3) $\operatorname{dim} V_{\Pi}(k)=\operatorname{deg} g_{\Pi}$.

Proof. We observed that the set $V_{\Pi}(k)$ is a locally closed subset of the affine variety $H_{\alpha}^{\beta}(k)$. Note that the sets $\operatorname{Aut}_{\mathcal{N}}\left(N_{\alpha}(k)\right), \operatorname{Aut}_{\mathcal{S}}\left(M_{\Pi}(k)\right)$ are locally closed subsets of the affine varieties $\operatorname{End}_{k}\left(N_{\alpha}(k)\right), \operatorname{End}_{k}\left(N_{\alpha}(k), N_{\beta}(k), f\right)$, respectively. Applying Lemma 5.1, we prove that $V_{\Pi}(k)$, $\operatorname{Aut}_{\mathcal{N}}\left(N_{\alpha}(k)\right)$, $\operatorname{Aut}_{\mathcal{S}}\left(M_{\Pi}(k)\right)$ are closed under 
the Frobenius automorphism $\sigma$. Consider the subset $\operatorname{Aut}_{\mathcal{N}}\left(N_{\alpha}(k)\right)$ of $\operatorname{End}_{k}\left(N_{\alpha}(k)\right)$. Note that a matrix $X$ is in $\operatorname{Aut}_{\mathcal{N}}\left(N_{\alpha}(k)\right)$ if and only if $\operatorname{det} X \neq 0$ and $X \cdot A=A \cdot X$, where the matrix $A$ has entries 0 or 1 (apply Lemma 5.1). Therefore $\operatorname{det} X^{(p)} \neq$ 0 and $X^{(p)} A=(X \cdot A)^{(p)}=(A \cdot X)^{(p)}=A \cdot X^{(p)}$. This proves that $X^{(p)}$ is in $\operatorname{Aut}_{\mathcal{N}}\left(N_{\alpha}(k)\right)$. Similarly, we prove that $\operatorname{Aut}_{\mathcal{S}}\left(M_{\Pi}(k)\right)$ is closed under $\sigma$. By Lemma 5.1 $X$ is in $V_{\Pi}(k)$ if and only if there exists a matrix $Y \in V_{\Pi}(k)$ with entries 1 and 0 and an invertible matrix $A$ such that $X=A \cdot Y \cdot A^{-1}$. Then $X^{(p)}=\left(A \cdot Y \cdot A^{-1}\right)^{(p)}=A^{(p)} \cdot Y \cdot\left(A^{(p)}\right)^{-1}$. This shows that $X^{(p)}$ is in $V_{\Pi}(k)$.

Therefore by Proposition 5.3 .

$$
g_{\Pi}(q) \approx c q^{\operatorname{dim} G_{k}(\Pi)} .
$$

Finally,

$$
\operatorname{dim} V_{\Pi}(k)=\operatorname{deg} g_{\Pi} .
$$

The remaining statements follow in a similar way.

Proposition 5.6. Let $\alpha, \beta$ be partitions and let $M_{\Pi}(k)=\left(N_{\alpha}(k), N_{\beta}(k), f\right)$ be an object in $\mathcal{S}_{2}(k)$ with Klein tableau $\Pi$. Then

$$
\operatorname{dim} V_{\Pi}(k)=\operatorname{deg} g_{\Pi}
$$

for any algebraically closed field $k$.

Proof. Let $k$ be an arbitrary algebraically closed field. It is well known that

- $\operatorname{dim} \operatorname{Aut}_{\mathcal{N}}\left(N_{\alpha}(k)\right)=\operatorname{dim}_{k} \operatorname{End}_{\mathcal{N}}\left(N_{\alpha}(k)\right)$,

- $\operatorname{dim} \operatorname{Aut}_{\mathcal{S}}\left(M_{\Pi}(k)\right)=\operatorname{dim}_{k} \operatorname{End}_{\mathcal{S}}\left(M_{\Pi}(k)\right)$.

Developing Lemma 5.1, it is easy to see that the properties

- $\operatorname{dim}_{k} \operatorname{End}_{\mathcal{N}}\left(N_{\alpha}(k)\right)=n_{k}$,

- $\operatorname{dim}_{k} \operatorname{End}_{\mathcal{S}}\left(M_{\Pi}(k)\right)=m_{k}$

can be written as first order formulae in the language of rings [4, Chapter 10]. By Proposition 5.5, for any $k=\overline{\mathbb{F}_{p}}$ we have $n_{k}=\operatorname{deg} a_{\alpha}$ and $m_{k}=\operatorname{deg} a_{\Pi}$. Therefore these numbers do not depend on the prime number $p$. The Characteristic Transfer Principle [4, Theorem 1.14] and Proposition 5.5]applied to these formulae yield that for any algebraically closed field $k$ we have

- $\operatorname{dim} \operatorname{Aut}_{\mathcal{N}}\left(N_{\alpha}(k)\right)=\operatorname{deg} a_{\alpha}$,

- $\operatorname{dim} \operatorname{Aut}_{\mathcal{S}}\left(M_{\Pi}(k)\right)=\operatorname{deg} a_{\Pi}$.

Then formula (5.2) proves that

$$
\operatorname{dim} V_{\Pi}(k)=\operatorname{deg} g_{\Pi} .
$$

We can now complete the proof of Theorem 1.3

Proof. For the proof that $\operatorname{dim} V_{\Pi}(k)=\operatorname{deg} h_{\alpha, \gamma}^{\beta}+\operatorname{deg} a_{\alpha}-x(\Delta)$, we have already seen that $\operatorname{dim} V_{\Pi}(k)=\operatorname{deg} g_{\Pi}$ (Proposition [5.6). Since each image of an embedding $N_{\alpha}(q) \rightarrow N_{\beta}(q)$ can be realized by $a_{\alpha}(q)$ many monomorphisms, we deduce

$$
g_{\Gamma}=h_{\Gamma} \cdot a_{\alpha}, \quad \text { and } \quad g_{\Pi}=h_{\Pi} \cdot a_{\alpha} .
$$

We recall from [12, II, (1.6)] that $\operatorname{deg} a_{\alpha}=|\alpha|+2 n(\alpha)$. The equality $\operatorname{deg} h_{\Pi}=$ $\operatorname{deg} h_{\Gamma}-x(\Pi)$ is shown in [8]: In Corollary 1, the Hall polynomial is computed as

$$
h_{\Pi}(q)=q^{\operatorname{deg} h_{\Gamma}} f\left(\Pi, \frac{1}{q}\right) .
$$


According to the paragraph leading to [8, Definition 3.5], $q^{x(\Pi)} f\left(\Pi, \frac{1}{q}\right)$ is a polynomial in $\frac{1}{q}$ with constant coefficient 1 . Then $q^{\operatorname{deg} h_{\Gamma}} f\left(\Pi, \frac{1}{q}\right)$ is a monic polynomial in $q$ of degree $\operatorname{deg} h_{\Gamma}-x(\Pi)$. It remains to observe that $\operatorname{deg} h_{\Gamma}=\operatorname{deg} h_{\alpha, \gamma}^{\beta}=$ $n(\beta)-n(\alpha)-n(\gamma)$ [8, Corollary 2, p. 77], and that $x(\Delta)=x(\Pi)($ Lemma 2.2) .

5.3. Properties of the partial order $\leq_{\text {arc }}$. Let $\mathcal{K}(\Gamma)$ be the set of all Klein tableaux refining an LR-tableau $\Gamma$ with entries at most two, and $\mathcal{K}(\alpha, \beta, \gamma)$ the set of all Klein tableaux of partition type $(\alpha, \beta, \gamma)$ with $\alpha_{1} \leq 2$. We describe properties of the posets $\mathcal{K}(\Gamma)=\left(\mathcal{K}(\Gamma), \leq_{\text {arc }}\right)$ and $\mathcal{K}(\alpha, \beta, \gamma)=\left(\mathcal{K}(\alpha, \beta, \gamma), \leq_{\text {arc }}\right)$.

Theorem 5.7. Let $\Gamma$ be an LR tableau with entries at most two.

(1) In the poset $\mathcal{K}(\Gamma)$ there exists exactly one minimal element: the dominant Klein tableau.

(2) In the poset $\mathcal{K}(\Gamma)$ there exists exactly one maximal element.

(3) In the poset $\mathcal{K}(\alpha, \beta, \gamma)$ there exists exactly one maximal element.

(4) The set of minimal elements of the poset $\mathcal{K}(\alpha, \beta, \gamma)$ consists of the minimal elements of the posets $\mathcal{K}\left(\Gamma_{1}\right), \ldots, \mathcal{K}\left(\Gamma_{s}\right)$, where $\Gamma_{1}, \ldots, \Gamma_{s}$ are all the $L R$ tableaux of type $(\alpha, \beta, \gamma)$.

Proof. 1. Assume that $\Pi \in \mathcal{K}(\Gamma)$ is not dominant. Recalling from Section 2.1 how the dominant Klein tableau is obtained, it follows that there exists a row $m$ in $\Pi$ with a symbol $22_{r}$, where $r$ is not the largest available subscript. Choose $m$ minimal with this property and denote by $r^{\prime}$ the largest available subscript that could be assigned to 2 in this row. Therefore in the arc diagram corresponding to $\Pi$ we have:

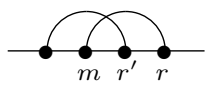

or

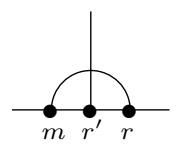

This proves that $\Pi$ is not arc-minimal. Then only the dominant tableau can be arc-minimal.

2. We prove that the unique arc-maximal $\Pi \in \mathcal{K}(\Gamma)$ is defined as follows: From an LR-tableau $\Gamma$ one can obtain a Klein tableau $\Pi$ by working through the entries in $\Gamma$ row by row (starting at the top) and assigning to each symbol $\ell \geq 2$ the smallest available subscript (due to the lattice permutation property, there is always a subscript available).

Assume that $\Pi \in \mathcal{K}(\Gamma)$ does not satisfy this condition. Then there exists a row $m$ with symbol $22_{r}$, where $r$ is not the smallest available subscript. Choose $m$ minimal with this property and denote by $r^{\prime}$ the smallest available subscript that could be assigned to 2 in this row. Therefore in the arc diagram corresponding to $\Pi$ we have:

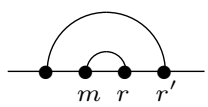

or

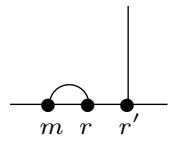

This proves that $\Pi$ is not arc-maximal.

3. Define $\Pi \in \mathcal{K}(\alpha, \beta, \gamma)$ as follows. Choose an LR-tableau $\Gamma \in \mathcal{K}(\alpha, \beta, \gamma)$ such that the entries 2 are in the largest available rows. Let $\Pi$ be the arc-maximal element in $\mathcal{K}(\Gamma)$. It is easy to see that we cannot apply to $\Pi$ moves of types $(\mathbf{C})$, 
(D), because these moves sent the entries 2 to larger rows. Since $\Pi$ is the arcmaximal element in $\mathcal{K}(\Gamma)$, we cannot apply to $\Pi$ moves of types $(\mathbf{A}),(\mathbf{B})$. Therefore $\Pi$ is an arc-maximal element in $\mathcal{K}(\alpha, \beta, \gamma)$.

Assume that $\Pi^{\prime}$ is an element in $\mathcal{K}(\alpha, \beta, \gamma)$ such that in the LR-tableau $\Gamma^{\prime}$ corresponding to $\Pi^{\prime}$ there exists an entry 2 in the row $r$ that is not the largest possible. Therefore in the arc diagram corresponding to $\Pi^{\prime}$ we have:

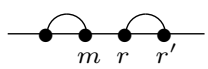

or

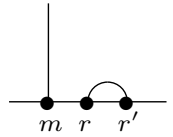

This proves that $\Pi^{\prime}$ is not arc-maximal.

4. Let $\Pi_{1}, \ldots, \Pi_{s}$ be the minimal elements in the posets $\mathcal{K}\left(\Gamma_{1}\right), \ldots, \mathcal{K}\left(\Gamma_{s}\right)$, respectively. Since the orbits $G_{\Pi_{1}}(k), \ldots, G_{\Pi_{s}}(k)$ have maximal dimension equal to $\operatorname{deg} g_{\alpha, \gamma}^{\beta}$ and since the arc-order is equivalent to the deg-order, the elements $\Pi_{1}, \ldots, \Pi_{s}$ are minimal in $\mathcal{K}(\alpha, \beta, \gamma)$. It is easy to see that they are the only minimal elements.

Example 1. Consider the following LR-tableau of type $(\alpha, \beta, \gamma)$, where $\alpha=$ $(2,2,1,1), \gamma=(4,3,2,2,1)$ and $\beta=(5,4,3,3,2,1)$ :

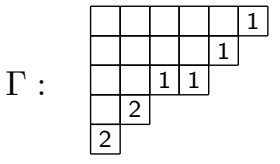

The arc diagrams $\Pi_{1}, \ldots, \Pi_{7}$ corresponding to $\Gamma$ are pictured in the Hasse diagram.

By [12, II.(4.1)], we have $\operatorname{deg} h_{\Gamma}=h_{\alpha, \gamma}^{\beta}=8$; with $\operatorname{deg} a_{\alpha}=20$ it follows that $\operatorname{deg} g_{\Gamma}=28$. We use Theorem 1.3 to compute $\operatorname{deg} g_{\Pi_{i}}=\operatorname{deg} g_{\Gamma}-x\left(\Pi_{i}\right)$, for $i=$ $1, \ldots, 7$. Note that

- the Klein tableau corresponding to $\Pi_{1}$ is dominant, so $x\left(\Pi_{1}\right)=0$ and $\operatorname{dim} G_{\Pi_{1}}=28$

- $x\left(\Pi_{2}\right)=1$ and $\operatorname{dim} G_{\Pi_{2}}=27$;

- $x\left(\Pi_{3}\right)=x\left(\Pi_{4}\right)=2$ and $\operatorname{dim} G_{\Pi_{3}}=\operatorname{dim} G_{\Pi_{4}}=26$;

- $x\left(\Pi_{5}\right)=3$ and $\operatorname{dim} G_{\Pi_{5}}=25$;

- $x\left(\Pi_{6}\right)=4$ and $\operatorname{dim} G_{\Pi_{6}}=24$

- $x\left(\Pi_{7}\right)=5$ and $\operatorname{dim} G_{\Pi_{7}}=23$

(compare Proposition [5.6).

Example 2. Consider the following triple of partitions $(\alpha, \beta, \gamma)$, where $\alpha=(2,1,1)$, $\gamma=(3,2,1)$ and $\beta=(4,3,2,1)$. There are three LR-tableaux of type $(\alpha, \beta, \gamma)$ :

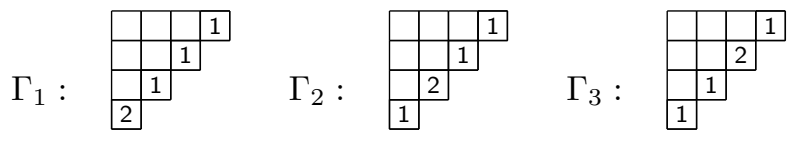

Note that $\Gamma_{1} \leq_{\text {part }} \Gamma_{2} \leq_{\text {part }} \Gamma_{3}$ and

$$
\mathcal{K}\left(\Gamma_{1}\right)=\left(\Pi_{1} \rightarrow \Pi_{4} \rightarrow \Pi_{6}\right), \mathcal{K}\left(\Gamma_{2}\right)=\left(\Pi_{2} \rightarrow \Pi_{5}\right), \mathcal{K}\left(\Gamma_{3}\right)=\left(\Pi_{3}\right),
$$

where $\Pi_{1}, \ldots, \Pi_{6}$ are as indicated in the Hasse diagram. 


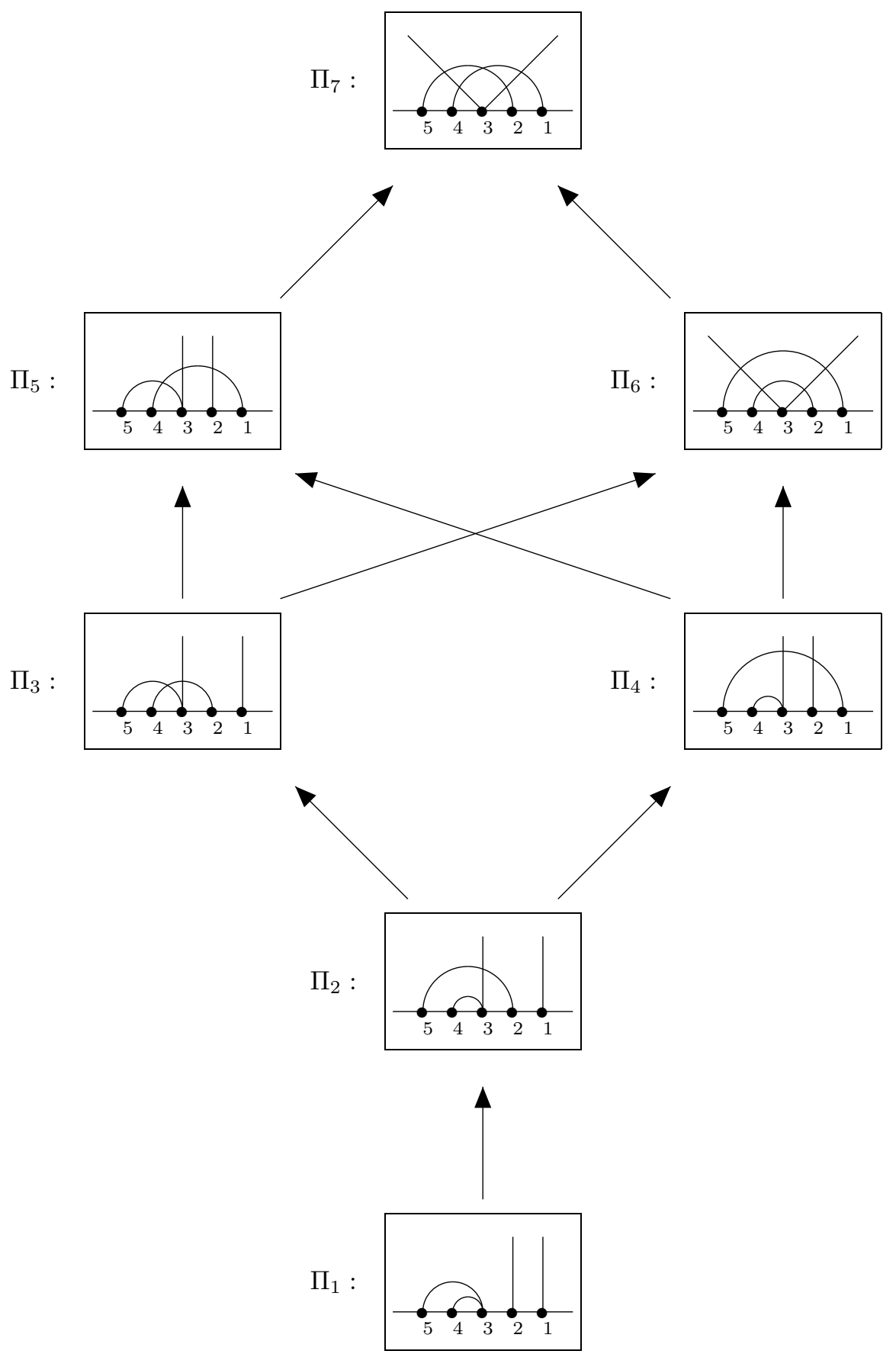

Figure 1. Hasse diagram of the poset $\mathcal{K}(\Gamma)$ from Example 1. 


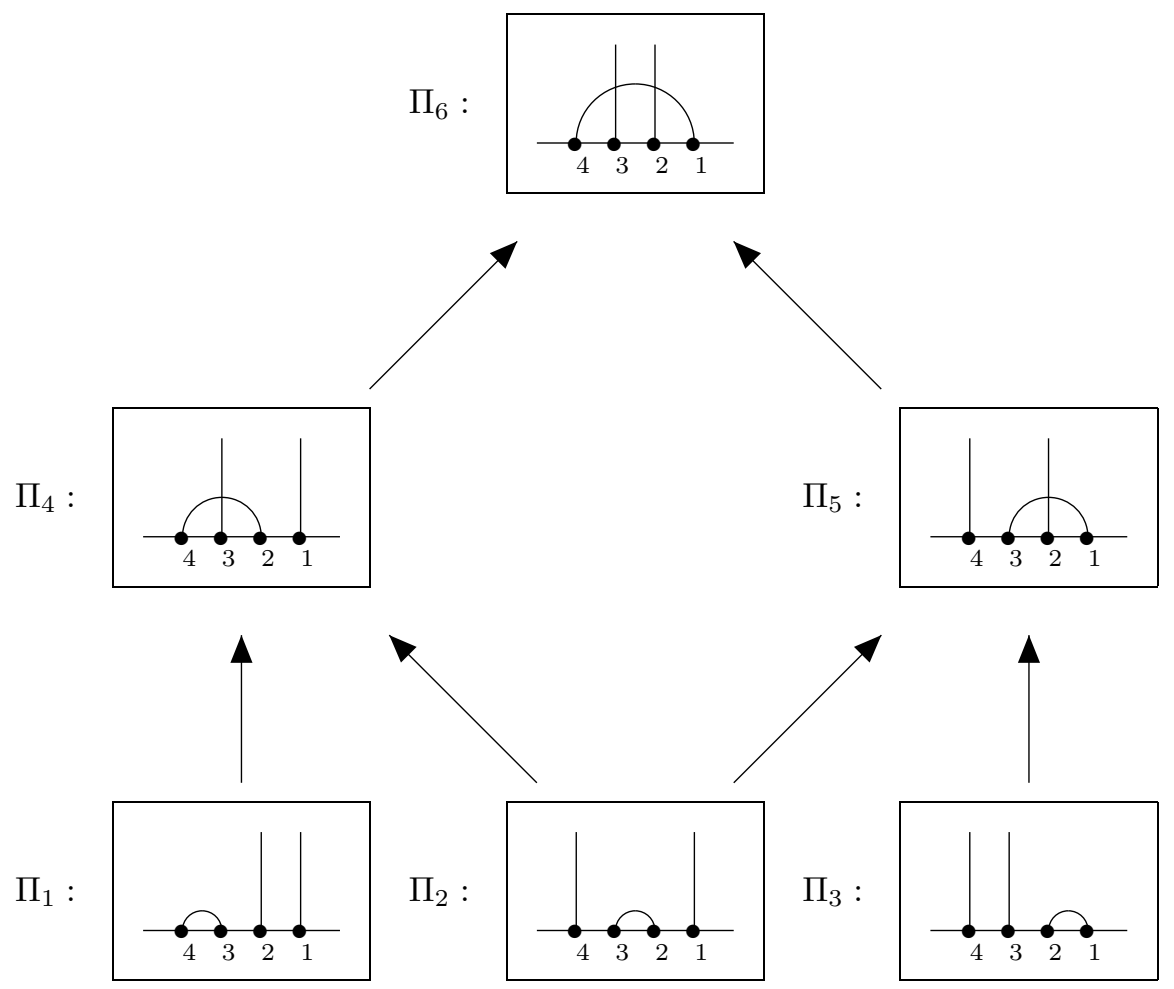

Figure 2. Hasse diagram of the poset $\mathcal{K}(\alpha, \beta, \gamma)$ from Example 2.

\section{ACKNOWLEDGEMENT}

The first named author wishes to thank Florida Atlantic University, where the authors started to write this paper, for the invitation. The second named author gratefully recognizes the invitation and support for his visit to Nicolaus Copernicus University in Torun during December 2011. Both authors want to thank Grzegorz Zwara for the discussion on the geometric part of this paper.

\section{DEDICATION}

The authors wish to dedicate this paper to Professor Daniel Simson on the occasion of his 70th birthday. Throughout our careers, we have known Professor Simson as a devoted scientist who has nurtured our interests towards attractive areas in algebra. We thank him for taking part in our mathematical development and for supporting us in a variety of ways.

\section{REFERENCES}

[1] Garrett Birkhoff, Subgroups of Abelian Groups, Proc. London Math. Soc. S2-38, no. 1, 385, DOI 10.1112/plms/s2-38.1.385. MR.1576323

[2] Klaus Bongartz, On degenerations and extensions of finite-dimensional modules, Adv. Math. 121 (1996), no. 2, 245-287, DOI 10.1006/aima.1996.0053. MR1402728(98e:16012) 
[3] Klaus Bongartz, Degenerations for representations of tame quivers, Ann. Sci. École Norm. Sup. (4) 28 (1995), no. 5, 647-668. MR.1341664 (96i:16020)

[4] Christian U. Jensen and Helmut Lenzing, Model-theoretic algebra with particular emphasis on fields, rings, modules, Algebra, Logic and Applications, vol. 2, Gordon and Breach Science Publishers, New York, 1989. MR.1057608 (91m:03038)

[5] Stanisław Kasjan, Representation-directed algebras form an open scheme, Colloq. Math. 93 (2002), no. 2, 237-250, DOI 10.4064/cm93-2-3. MR1930801(2003h:16022)

[6] Stanisław Kasjan and Justyna Kosakowska, On Lie algebras associated with representation-directed algebras, J. Pure Appl. Algebra 214 (2010), no. 5, 678-688, DOI 10.1016/j.jpaa.2009.07.012. MR2577675(2011d:17037)

[7] T. Klein, The multiplication of Schur-functions and extensions of p-modules, J. London Math. Soc. 43 (1968), 280-284. MR0228481 (37 \#4061)

[8] T. Klein, The Hall polynomial, J. Algebra 12 (1969), 61-78. MR0236260 (38 \#4557)

[9] Justyna Kosakowska, Degenerations in a class of matrix varieties and prinjective modules, J. Algebra 263 (2003), no. 2, 262-277, DOI 10.1016/S0021-8693(03)00173-X. MR.1978651 (2004d:16027)

[10] H. Kraft and Ch. Riedtmann, Geometry of representations of quivers, Representations of algebras (Durham, 1985), London Math. Soc. Lecture Note Ser., vol. 116, Cambridge Univ. Press, Cambridge, 1986, pp. 109-145. MR897322 (88k:16028)

[11] Serge Lang and André Weil, Number of points of varieties in finite fields, Amer. J. Math. 76 (1954), 819-827. MR0065218 (16,398d)

[12] I. G. Macdonald, Symmetric functions and Hall polynomials, 2nd ed., Oxford Mathematical Monographs, The Clarendon Press Oxford University Press, New York, 1995. With contributions by A. Zelevinsky; Oxford Science Publications. MR 1354144 (96h:05207)

[13] Christine Riedtmann, Degenerations for representations of quivers with relations, Ann. Sci. École Norm. Sup. (4) 19 (1986), no. 2, 275-301. MR868301 (88b:16051)

[14] Claus Michael Ringel, Hall algebras, Topics in algebra, Part 1 (Warsaw, 1988), Banach Center Publ., vol. 26, PWN, Warsaw, 1990, pp. 433-447. MR.1171248 (93f:16027)

[15] Claus Michael Ringel and Markus Schmidmeier, Invariant subspaces of nilpotent linear operators. I, J. Reine Angew. Math. 614 (2008), 1-52, DOI 10.1515/CRELLE.2008.001. MR2376281(2009d:16016)

[16] Markus Schmidmeier, Bounded submodules of modules, J. Pure Appl. Algebra 203 (2005), no. 1-3, 45-82, DOI 10.1016/j.jpaa.2005.02.003. MR2176651(2006j:16022)

[17] Markus Schmidmeier, Hall polynomials via automorphisms of short exact sequences, Algebr. Represent. Theory 15 (2012), no. 3, 449-481, DOI 10.1007/s10468-010-9250-6. MR2912467

[18] Daniel Simson, Representation types of the category of subprojective representations of a finite poset over $K[t] /\left(t^{m}\right)$ and a solution of a Birkhoff type problem, J. Algebra 311 (2007), no. 1, 1-30, DOI 10.1016/j.jalgebra.2007.01.029. MR2309875 (2009b:16040)

[19] Grzegorz Zwara, Degenerations for representations of extended Dynkin quivers, Comment. Math. Helv. 73 (1998), no. 1, 71-88, DOI 10.1007/s000140050046. MR1610587(99b:16021)

Faculty of Mathematics and Computer Science, Nicolaus Copernicus University, ul. Chopina 12/18, 87-100 Toruń, Poland

E-mail address: justus@mat.umk.pl

Department of Mathematical Sciences, Florida Atlantic University, 777 Glades Road, Boca Raton, Florida 33431

E-mail address: markus@math.fau.edu 